\title{
Selective Synthesis of Ortho-Substituted 2-Aryl-3-Phenyl-1,3-Thiazolidin-4-one Sulfoxides and Sulfones by S-Oxidation with Oxone ${ }^{\circledR}$
}

\author{
Kevin C. Cannon ${ }^{1}$, Alaa Alkurdi ${ }^{1}$, Humayra Himel ${ }^{1}$, Iryna Kurochka ${ }^{1}$, Sabrina Liu ${ }^{1}$, Miguel Costa ${ }^{1}$, Brynn Sundberg $^{2} \&$ \\ Anthony F. Lagalante ${ }^{2}$ \\ ${ }^{1}$ Department of Chemistry, Pennsylvania State University, Abington Campus, Abington, Pennsylvania, USA \\ ${ }^{2}$ Department of Chemistry, Villanova University, Villanova, Pennsylvania, USA \\ Correspondence: Kevin C. Cannon, Department of Chemistry, 1600 Woodland Road, Pennsylvania State University, \\ Abington Campus, Abington, Pennsylvania 19001, USA. E-mail: kcc10@psu.edu
}

Received: September 18, 2017 Accepted: October 18, 2017 Online Published: October 22, 2017

doi:10.5539/ijc.v9n4p87

URL: https://doi.org/10.5539/ijc.v9n4p87

\section{Abstract}

$S$-oxidation of 2-aryl-3-phenyl-1,3-thiazolidin-4-ones with Oxone ${ }^{\circledR}$ was investigated. For all compounds evaluated, selective oxidation to the sulfoxide was realized using 3 equivalents of Oxone ${ }^{\circledR}$ at room temperature. Attempts to selectively prepare the sulfones of ortho-substituted 2-aryl-3-phenyl-1,3-thiazolidin-4-ones at high temperature by increasing the equivalents of Oxone $^{\circledR}$ used were typically unsuccessful. These results contrast significantly with ortho-substituted 2-aryl-3-cyclohexyl-1,3-thiazolidin-4-ones evaluated previously. The extent of this selectivity was affected by the substituent and its position on the $\mathrm{C} 2$ aromatic ring. The ratio of the sulfoxide and sulfone products was quantified by isolating the products by liquid chromatography.

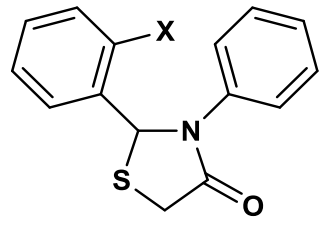

thiazolidin-4-one

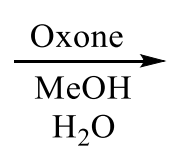

$\mathrm{H}_{2} \mathrm{O}$

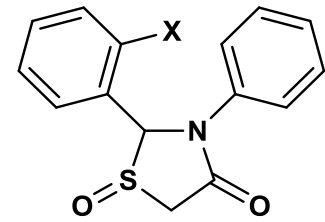

sulfoxide
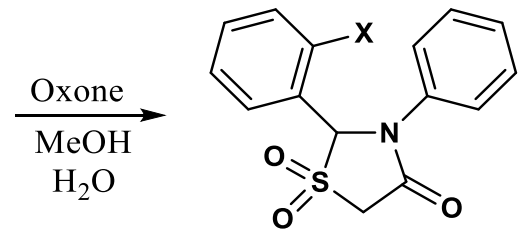

sulfone

Keywords: thiazolidin-4-ones, Oxone, sulfoxide, sulfone

\section{Introduction}

1,3-Thiazolidin-4-ones, also known as thiazolidin-4-ones, are known to have a very wide range of biological activity.(Suryawanshi et al., 2017)(Kaushal \& Kaur, 2016)(Kumar, Kumar, Mundlia, Pradhan \& Malik, 2015)(Tripathi et al., 2014)(Jain, Vaidya, Ravichandran, Kashaw, \& Agrawal, 2012)(Abhinit, Ghodke \& Pratima, 2009)(Hamama, Ismail, Shaaban \& Zoorob, 2008)(Singh, Parmar, Raman, Virgil \& Stenberg, 1981)(Brown, 1961), so much that some have referred to it as a "magic moiety" or "wonder nucleus"(Jain et al., 2012). The $S$-oxides may show enhanced activity; for example, Miller and coworkers converted one 4-thiazolidinone to its sulfoxide and sulfone and reported that the oxides showed greater activity against some cancer cell lines than the sulfide.(Gududuru, Hurh, Dalton \& Miller, 2004) Thiazolidin-4-ones have been oxidized to sulfoxides with peracetic acid (Surrey, 1967), $\mathrm{Na}_{5} \mathrm{IO}_{6}(\mathrm{Smith}$, Lee \& Cragoe, 1977), chloramine T (Omar, El-Kharmy \& Sharif, 1981), $\mathrm{NaIO}_{4}$ (Lee, Yergatian, Crowther \& Downie, 1990), Oxone ${ }^{\circledR}$ (one example)(Rozwadowska, Sulima \& Gzella, 2002), and $m$-CPBA (Rozwadowska \& Sulima, 2003). Oxidation from sulfide to sulfoxide makes the sulfur a chiral center, and produces cis and trans diastereomers with relation to C-2 (Rozwadowska et al., 2002) (Colombo et al., 2008). The stereocenters, however, may be configurationally unstable (Rozwadowska et al., 2002). Oxidation of thiazolidin-4-ones to sulfones has been accomplished with $\mathrm{H}_{2} \mathrm{O}_{2} / \mathrm{Ac}_{2} \mathrm{O} / \mathrm{AcOH}$ (Troutman \& Long, 1948), and $\mathrm{KMnO}_{4}$ (Surrey, 1948).

Oxone ${ }^{\circledR}$, a mixture of potassium sulfates $\left(2 \mathrm{KHSO}_{5} / 1 \mathrm{~K}_{2} \mathrm{SO}_{4} / 1 \mathrm{KHSO}_{4}\right)$, is a very desirable material to use because it is a "green" reagent which is inexpensive, safe, and easy to use (Yu et al., 2012) (Hussain, Green \& Ahmed, 2013). It has been 
used as a chemoselective reagent for the oxidation of sulfides to either sulfoxides (Trost \& Curran, 1981) (Yu et al., 2012) (Webb, 1994) (Madesclaire, 1986) or sulfones (Trost \& Curran, 1981) (Yu et al., 2012) (Webb, 1994). Selectivity toward the sulfoxide or sulfone has been shown to depend on the amount of Oxone ${ }^{\circledR}$ used, the temperature, and the solvent (Trost \& Curran, 1981) (Yu et al., 2012) (Webb, 1994).

Although there are ample examples of Oxone ${ }^{\circledR}$-based oxidations of sulfides, there is little data related specifically to the oxidation of thiazolidin-4-ones. Rozwadowska et al., (2002) reported a single example of oxidation of a thiazolidin-4-one to its sulfoxide with this reagent. Convenient synthetic access to thiazolidin-4-one $S$-oxides would encourage further biological and pharmaceutical evaluation of these compounds, which prompted our current investigation. We have previously reported the reaction of ortho-substituted 2-aryl-3-cyclohexyl-1,3-thiazolidin-4-ones with Oxone ${ }^{\circledR}$ (Cannon et al., 2015). For all ten compounds previously evaluated, selective oxidation to the sulfoxide was realized using 3 equivalents of Oxone ${ }^{\circledR}$ at room temperature, and subsequent oxidation to the sulfone was not observed under these reaction conditions after 25 hours. Alternatively, the sulfones were prepared with variable selectively at higher temperature (refluxing aqueous methanol) by increasing the equivalents of Oxone ${ }^{\circledR}$ used to 8.(Scheme 1) The extent of this selectivity was affected by the substituent on the $\mathrm{C} 2$ aromatic ring; sulfones were produced exclusively when the substituent (X) was $\mathrm{OCH}_{3}, \mathrm{OCH}_{2} \mathrm{CH}_{3}$, or $\mathrm{NO}_{2}$. Sulfone formation was significantly favored when the substituent was $\mathrm{CH}_{3}$ or $\mathrm{H}$. Slight preference for sulfone versus sulfoxide formation was observed when the substituent $(\mathrm{X})$ was $\mathrm{CF}_{3}$, but preference for sulfoxide formation was observed for halide substituents ( $\mathrm{X}=\mathrm{F}$ or $\mathrm{Br}$ ). No clear pattern of reactivity was realized based on the substituents' electronic properties or size.
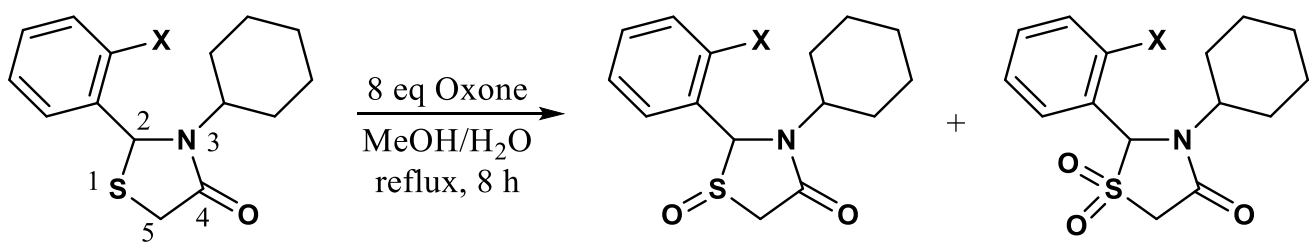

$$
\mathrm{X}=\mathrm{H}, \mathrm{F}, \mathrm{Cl}, \mathrm{Br}, \mathrm{CH}_{3}, \mathrm{CF}_{3}, \mathrm{OCH}_{3}, \mathrm{OCH}_{2} \mathrm{CH}_{3}, \mathrm{NO}_{2}, 1 \text {-naphthyl }
$$

Scheme 1. Oxidation of thiazolidin-4-ones using high temperature Oxone ${ }^{\circledR}$-based reaction conditions

A subsequent evaluation of $S$-oxidation of meta- and para-substituted 3-aryl-2-phenyl-1,3-thiazolidin-4-ones with Oxone ${ }^{\circledR}$ likewise demonstrated selective oxidation to the sulfoxide using 3 equivalents of Oxone ${ }^{\circledR}$ at room temperature (Cannon, et al., 2017). At high temperature oxidations using 8 equivalents of Oxone ${ }^{\circledR}$, the extent of selectivity in sulfone formation was affected by the substituent and its location on the N3 aromatic ring. The only clear substituent/reactivity correlation evidenced was better selectivity of sulfone versus sulfoxide formation when electron donating substituents $\left(\mathrm{X}=\mathrm{CH}_{3}\right.$ and $\mathrm{OCH}_{3}$ ) were in the para position; for all other substituents, meta substitution showed higher sulfone selectivity versus para substitution. Unlike the ortho-substituted 2-aryl-3-cyclohexyl-1,3-thiazolidin-4-ones, exclusive formation of sulfone versus sulfoxide was never realized by Oxone ${ }^{\circledR}$ oxidation, and overall yields decreased significantly. Exclusive sulfone formation was best achieved using 2 equivalents of $\mathrm{KMnO}_{4}$.

In this study, we report the high temperature oxidation of a series of ortho-substituted 2-aryl-3-phenyl-1,3-thiazolidin-4-ones with Oxone ${ }^{\circledR}$ to determine if changing the N3 substituent from cyclohexyl to phenyl affects oxidative selectivity. We also report room temperature oxidations of this series of thiazolidin-4-ones with Oxone ${ }^{\circledR}$ and $\mathrm{KMnO}_{4}$ to ascertain the scope and selectivity of the Oxone ${ }^{\circledR}$ oxidations.

\section{Results and Discussion}

\subsection{Preparation of Ortho-Substituted Thiazolidin-4-Ones}

The ortho-substituted 2-aryl-3-phenyl-1,3-thiazolidin-4-ones 1a-h used in this evaluation were prepared by sequential condensation reactions.(Tierney et al., 2005) The compounds were prepared by condensation of an ortho-substituted benzaldehyde with aniline to produce an imine intermediate, followed by condensation with thioglycolic acid (Scheme 2). Reaction progress in both steps was monitored by the collection of water in a Dean-Stark trap. 


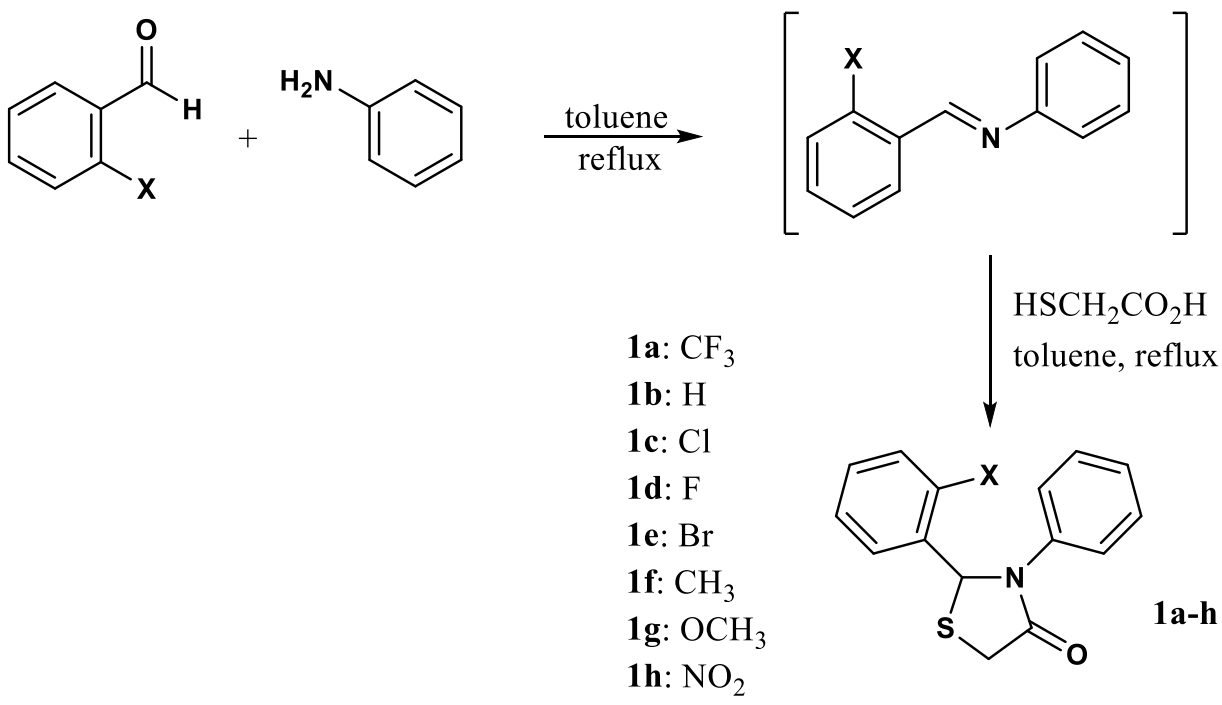

Scheme 2. Synthesis of ortho-substituted 3-aryl-2-phenyl-1,3-thiazolidin-4-ones 1a-h

\subsection{Low Temperature Oxone ${ }^{\circledR}$ Oxidations of Thiazolidin-4-Ones}

Exclusive formation of sulfoxide compounds $\mathbf{2 a - 2} \mathbf{h}$ was realized by performing the oxidation at room temperature with a 3 equivalents of Oxone ${ }^{\circledR}$ (Scheme 3). The reaction time for sulfoxide formation at $1 \mathrm{~h}$ insured complete conversion of the thiazolidin-4-ones which was confirmed by thin layer chromatography (TLC). Results are summarized in Table 1. Clearly, low temperature oxidation with a reduced number of Oxone ${ }^{\circledR}$ equivalents favors sulfoxide formation as was previously observed for the 3-cyclohexyl-thiazolidin-4-ones (Cannon et al., 2015).

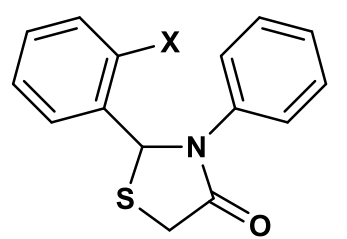

1a-h

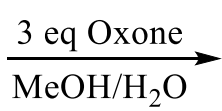

$\mathrm{rt}, 1 \mathrm{~h}$<smiles>[X]c1ccccc1C1N(c2ccccc2)C(=O)CS1=O</smiles>

2a-h

Scheme 3. Selective low temperature Oxone ${ }^{\circledR}$ oxidation of thiazolidin-4-ones to sulfoxides

Table 1. Ortho-substituted 2-aryl-3-phenyl-1,3-thiazolidin-4-one sulfoxides $\mathbf{2 a - 2 h}$ synthesized according to Scheme 3

Product \% Yield Melting Point $\left({ }^{\circ} \mathrm{C}\right)$

$\mathrm{R}_{\mathrm{f}}$

(3:1 cyclohexane:EtOAc)

\begin{tabular}{lccc}
\hline $\mathbf{2 a}, \mathrm{X}=\mathrm{CF}_{3}$ & 98 & oil & 0.199 \\
$\mathbf{2 b}, \mathrm{X}=\mathrm{H}$ & 96 & $163-164$ & 0.159 \\
$\mathbf{2 c}, \mathrm{X}=\mathrm{Cl}$ & 99 & $60-62$ & 0.158 \\
$\mathbf{2 d}, \mathrm{X}=\mathrm{F}$ & 99 & $179-180$ & 0.098 \\
$\mathbf{2 e}, \mathrm{X}=\mathrm{Br}$ & 83 & $154-155$ & 0.135 \\
$\mathbf{2 f}, \mathrm{X}=\mathrm{CH}_{3}$ & 99 & $172-174$ & 0.154 \\
$\mathbf{2 g}, \mathrm{X}=\mathrm{OCH}_{3}$ & 99 & $184-185$ & 0.084 \\
$\mathbf{2 h}, \mathrm{X}=\mathrm{NO}_{2}$ & 98 & $78-79$ & 0.167 \\
\hline
\end{tabular}

2.3 High Temperature Oxone ${ }^{\circledR}$ Oxidations of Thiazolidin-4-Ones

Compounds 1a-h were oxidized according to the high temperature Oxone ${ }^{\circledR}$-based reaction conditions that had been previously optimized for 2-aryl-3-cyclohexyl-1,3-thiazolidin-4-ones (Cannon et al., 2015) to compare selective formation of sulfones between the two sets of thiazolidin-4-ones (Scheme 4). Results are presented in Table 2. 


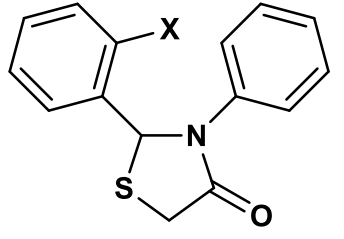

1a-h

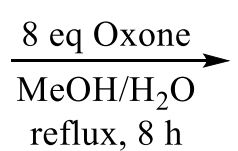

Scheme

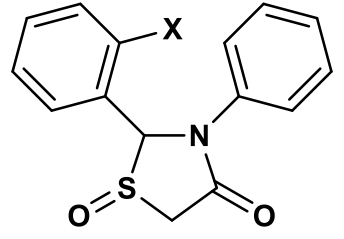

2a-h

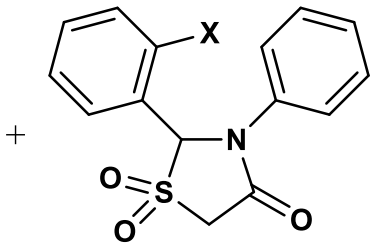

3a-h

The ratio indicates the relative amounts of sulfoxide to sulfone isolated by chromatography. Also included are the ratios observed for the respective ortho-substituted 2-aryl-3-cyclohexyl-1,3-thiazolidin-4-ones. (Cannon et al., 2015)

Table 2. Oxidation of thiazolidin-4-ones 1a-h using high temperature Oxone ${ }^{\circledR}$-based reaction conditions

\begin{tabular}{|c|c|c|}
\hline $\begin{array}{l}\text { Thiazolidin-4-one } \\
\text { (1) }\end{array}$ & $\begin{array}{c}\text { Sulfoxide:Sulfone (2:3) } \\
\text { (Total Yield) }\end{array}$ & $\begin{array}{l}\text { Sulfoxide:Sulfone and } \\
\text { Total Yield for the } \\
\text { respective } \\
N \text {-Cyclohexyl-substituted } \\
\text { Thiazolidin-4-one }\end{array}$ \\
\hline 1a, $\mathrm{X}=\mathrm{CF}_{3}$ & $1.0: 1.5(89 \%)$ & 1.0: $1.7(89 \%)$ \\
\hline 1b, $X=H$ & $2.0: 1.0(76 \%)$ & $1.0: 3.2(93 \%)$ \\
\hline 1c, $\mathrm{X}=\mathrm{Cl}$ & $1.1: 1.0(62 \%)$ & $1.0: 1.2(98 \%)$ \\
\hline 1d, $X=F$ & $1.0: 1.1(66 \%)$ & $2.0: 1.0(95 \%)$ \\
\hline 1e, $\mathrm{X}=\mathrm{Br}$ & $1.0: 1.1(62 \%)$ & $2.1: 1.0(88 \%)$ \\
\hline 1f, $\mathrm{X}=\mathrm{CH}_{3}$ & $1.0: 1.3(80 \%)$ & $1.0: 4.9(75 \%)$ \\
\hline 1g, $\mathrm{X}=\mathrm{OCH}_{3}$ & $1.1: 1.0(81 \%)$ & Sulfone only (98\%) \\
\hline 1h, $\mathrm{X}=\mathrm{NO}_{2}$ & 7.6:1.0 (72\%) & Sulfone only (94\%) \\
\hline
\end{tabular}

Results in Table 2 show that sulfone formation significantly varied according to the substituent on N3. Only four of the eight $N$-phenyl-substituted thiazolidin-4-ones $\mathbf{1 a}-\mathbf{1 h}$ demonstrated a preference for sulfone formation, and the highest preference was an anemic $20 \%$ excess observed for $1 \mathbf{a}\left(\mathrm{X}=\mathrm{CF}_{3}\right)$. In contrast, the $N$-cyclohexyl-substituted thiazolidin-4-ones (Cannon, et al., 2015) demonstrated preferential sulfone formation for six of the eight compounds indicated, and exclusive sulfone formation was observed for both the corresponding methoxy and nitro derivatives. Even more interesting is that no substitution/reactivity correlations exist between the two sets of data. Preferences for sulfoxide versus sulfone formation flip for six of the eight aryl substituents, with the most significant changes in preference being observed for $\mathbf{1 g}$ and $\mathbf{1 h}$. The corresponding $N$-cyclohexyl-substituted thiazolidin-4-ones previously had demonstrated exclusive sulfone formation, while $\mathbf{1 g}$ and $\mathbf{1 h}$ showed the third and first highest preference for sulfoxide formation, respectively, in the current evaluation.

Overall, the total oxidation yields are lower for the 2-aryl-3-phenyl-1,3-thiazolidin-4-ones (average yield = 74\%) versus similarly substituted 2-aryl-3-cyclohexyl-1,3-thiazolidin-4-ones (average yield $=91 \%$ ) despite reactant conversions of $100 \%$ for all thiazolidin-4-ones evaluated. In a prior publication, diaryl thiazolidin-4-one sulfones were stable under reaction conditions ( 8 Oxone ${ }^{\circledR}$ equivalents in refluxing aqueous methanol for $8 \mathrm{~h}$ ) as determined by TLC and sulfone recovery (Cannon, et al., 2017). Therefore, product decomposition cannot account for either the lower observed oxidation yields or the relatively higher sulfoxide formation.

To further test the potential effect of $\mathrm{C} 2$ aryl substitution on sulfone formation and yields, a small group of para-substituted 2-aryl-3-phenyl-1,3-thiazolidin-4-ones (4a-4d) was evaluated under the same reaction conditions for high temperature oxidation (Scheme 5). The para-substituted and ortho-substituted thiazolidin-4-ones should have similar electronic properties, but the para-substituted thiazolidin-4-ones lack potential steric contributions associated with ortho substitution. Results summarized in Table 3 show better selectivity for sulfone formation and slightly improved oxidation yields for three of the four para-substituted thiazolidin-4-ones tested (4a, $\mathbf{4 b}$, and $\mathbf{4 d}$ ). Extensive decomposition of $\mathbf{4 c}$ during the high temperature Oxone ${ }^{\circledR}$ oxidation accounted for the low yield of oxidation products. Attempts to characterize the aldehyde- and alkene-containing decomposition by-products of $\mathbf{4 c}$ oxidation by NMR analysis were unsuccessful. Comparison of $\mathbf{1 d}$ (X = 
F), 1e $(X=B r)$, and $1 \mathbf{h}\left(X=\mathrm{NO}_{2}\right)$ to $\mathbf{4 a}, \mathbf{4 b}$, and $\mathbf{4 d}$ show that the position of the substituent in the $\mathrm{C} 2$ aryl ring significantly changed sulfone formation selectivity, and that this effect is probably sterically based. Room temperature oxidations of $\mathbf{4 a - 4 d}$ with Oxone ${ }^{\circledR}$ according to reaction conditions described previously produced the corresponding sulfoxides 5a-5d exclusively as observed for $\mathbf{1 a}-\mathbf{1 h}$. Data for these products can be found in the experimental section.<smiles>[X]c1ccc(C2SCC(=O)N2c2ccccc2)cc1</smiles>

4a-d

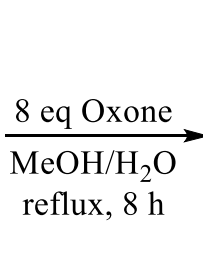<smiles>[X]c1ccc(C2N(c3ccccc3)C(=O)CS2=O)cc1</smiles>

5a-d<smiles>[X]c1ccc(C2N(c3ccccc3)C(=O)CS2(=O)=O)cc1</smiles>

6a-d

Scheme 5. High temperature Oxone ${ }^{\circledR}$ oxidation of para-substituted thiazolidin-4-ones

The ratio indicates the relative amounts of sulfoxide to sulfone isolated by chromatography.

Table 3. Oxidation of thiazolidin-4-ones 4a-d using high temperature Oxone ${ }^{\circledR}$-based reaction conditions

\begin{tabular}{lc}
\hline Thiazolidin-4-one & Sulfoxide:Sulfone (5:6) \\
(4) & (Total Yield)
\end{tabular}

\begin{tabular}{ll}
\hline $\mathbf{4 a}, \mathrm{X}=\mathrm{F}$ & $1.0: 2.0(79 \%)$ \\
$\mathbf{4 b}, \mathrm{X}=\mathrm{Br}$ & $1.0: 1.8(77 \%)$ \\
$\mathbf{4 c}, \mathrm{X}=\mathrm{OCH}_{3}$ & $1.0: 1.0(33 \%)$ \\
$\mathbf{4 d}, \mathrm{X}=\mathrm{NO}_{2}$ & $1.0: 1.8(82 \%)$
\end{tabular}

\section{4 $\mathrm{KMnO}_{4}$-based Oxidations of Thiazolidin-4-Ones}

Since Oxone ${ }^{\circledast}$ oxidations of 2-aryl-3-phenyl-1,3-thiazolidin-4-ones failed to produce sulfones with good selectivity, an alternative syntheses of the sulfones using aqueous $\mathrm{KMnO}_{4}$ was evaluated (Surrey, 1948). This method was applied to compounds 1a-1h to produce the corresponding sulfones (Scheme 6); results are summarized in Table 4. Although no measures were taken to optimize this reaction, the oxidation yields are overall significantly higher for the 2-aryl-3-phenyl-1,3-thiazolidin-4-ones (average yield=75\%) compared to the 2-aryl-3-cyclohexyl-1,3-thiazolidin-4-ones (average yield $=56 \%)$ previously synthesized by this method (Cannon et al., 2015).<smiles>[X]c1ccccc1C1SCC(=O)N1c1ccccc1</smiles>

1a-h

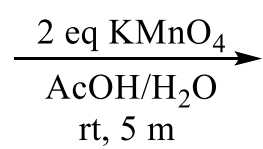

$\mathrm{rt}, 5 \mathrm{~m}$<smiles>[X]c1ccccc1C1N(c2ccccc2)C(=O)CS1(=O)=O</smiles>

3a-h

Scheme 6. $\mathrm{KMnO}_{4}$ oxidation of thiazolidin-4-ones to sulfones

Table 3. Ortho-substituted 2-aryl-3-phenyl-1,3-thiazolidin-4-one sulfones 3a-h synthesized according to Scheme 6

\begin{tabular}{lcc}
\hline \multicolumn{1}{c}{ Product } & \% Yield & Melting Point $\left({ }^{\circ} \mathrm{C}\right)$ \\
\hline 3a, $\mathrm{X}=\mathrm{CF}_{3}$ & 73 & oil \\
3b, $\mathrm{X}=\mathrm{H}$ & 76 & $178-180$ \\
$\mathbf{3 c}, \mathrm{X}=\mathrm{Cl}$ & 69 & oil \\
3d, $\mathrm{X}=\mathrm{F}$ & 76 & $159-160$ \\
$\mathbf{3 e}, \mathrm{X}=\mathrm{Br}$ & 91 & oil \\
$\mathbf{3 f}, \mathrm{X}=\mathrm{CH}_{3}$ & 70 & $143-144$ \\
$\mathbf{3 g}, \mathrm{X}=\mathrm{OCH}_{3}$ & 80 & $130-131$ \\
$\mathbf{3 4}, \mathrm{X}=\mathrm{NO}_{2}$ & 63 & $177-179$ \\
\hline
\end{tabular}


The $\mathrm{KMnO}_{4}$ oxidation procedure was likewise applied to the para-substituted 2-aryl-3-phenyl-1,3-thiazolidin-4-ones 4a-4d to produce the corresponding sulfones $\mathbf{6 a - 6 d}$. Data for these products can be found in the experimental section.

\section{Conclusion}

$S$-oxidation of 2-aryl-3-phenyl-1,3-thiazolidin-4-ones with Oxone ${ }^{\circledR}$ was dependent on the reaction temperature, equivalents of Oxone ${ }^{\circledR}$ used, the substituent, and the substituent's location on the $\mathrm{C} 2$ aromatic ring. For all thiazolidin-4-ones evaluated in this study, selective oxidation to the sulfoxide was realized by using 3 equivalents of Oxone ${ }^{\circledR}$ at room temperature. At high temperature using 8 equivalents of Oxone ${ }^{\circledR}$, oxidative yields the selectivity in sulfone formation varied according to the substituent although no clear substituent/reactivity correlation was evident based on the substituents' electronic properties. Location of the substituent on the $\mathrm{C} 2$ aromatic ring appeared to be important as well: para-substituted $\mathrm{C} 2$ aryls demonstrated slightly better sulfone formation selectivity than ortho-substituted $\mathrm{C} 2$ aryls according to the limited number of para-substituted compounds evaluated. Substitution at N3 of the thiazolidin-4-one ring also affects sulfone selectivity and yield. Unlike the Oxone ${ }^{\circledR}$ oxidation of ortho-substituted 2-aryl-3-cyclohexyl-1,3-thiazolidin-4-ones evaluated previously, exclusive formation of sulfone versus sulfoxide was never realized for 2-aryl-3-phenyl-1,3-thiazolidin-4-ones, and in fact most sulfone selectivity was poor. Exclusive sulfone formation was best achieved using 2 equivalents of $\mathrm{KMnO}_{4}$. Additionally, lower Oxone ${ }^{\circledR}$ oxidation yields were observed for $N$-phenyl thiazolidin-4-ones compared to previously evaluated $N$-cyclohexyl thiazolidin-4-ones.

\section{Experimental}

Reagent chemicals were obtained from commercial suppliers; Oxone ${ }^{\circledR}$ was purchased from Aldrich Chemical Company. TLC and chromatography plates (silica gel GF, 250 micron) were purchased from Analtech. Reagent grade solvents were used without further purification.

Most spectra were recorded on a Bruker 300 at $298 \mathrm{~K}$ observing ${ }^{1} \mathrm{H}$ and ${ }^{13} \mathrm{C}$ at 300.15 and $75.48 \mathrm{MHz}$, respectively. These samples were dissolved in $\mathrm{CDCl}_{3}$ at a concentration of $100 \mathrm{mg} / \mathrm{mL}$ using precision bore $5 \mathrm{~mm} \mathrm{NMR} \mathrm{tubes} \mathrm{supplied} \mathrm{by}$ Norell, Inc. The spectrometer was locked to either the deuterium or carbon resonance of $\mathrm{CDCl}_{3}$ and all chemical shifts were referenced to residual $\mathrm{CHCl}_{3}$.

Infrared spectra were obtained as an evaporated thin film on a sodium chloride plate (Janos Technology, Inc) on a Nicolet Nexus 670 spectrometer using 32 scans at a $2 \mathrm{~cm}^{-1}$ resolution. Mass spectra were recorded on a Varian $2100 \mathrm{G}$ ion trap mass spectrometer, fitted with a Varian 3900 gas chromatograph: column - Factor 4 VF- $5 \mathrm{~ms} 0.25 \mathrm{~mm}$ id, $30 \mathrm{~m}, 0.25 \mu \mathrm{m}$ film thickness, He carrier gas, $1.0 \mathrm{ml} / \mathrm{min}$ flow, $80^{\circ} \mathrm{C}$ for 1 minute isothermal $15^{\circ} \mathrm{C} / \mathrm{min}$ to $275^{\circ} \mathrm{C}$ then $275^{\circ} \mathrm{C}$ for 3 minutes isothermal, injector temp $250^{\circ} \mathrm{C}, 0 \mathrm{~min}, 1: 50$ split. Melting points are uncorrected; a Uni-Melt capillary melting point apparatus was used.

\subsection{Preparation of Substituted-2-Aryl-3-Phenyl-Thiazolidin-4-Ones}

All thiazolidine-4-ones were prepared using the procedure previously described. (Tierney et al., 2005) The para-substituted-2-aryl-3-phenyl-thiazolidin-4-ones $\mathbf{4 a - 4 d}$ and $\mathbf{1 b}$ had been previously characterized. (Tierney et al., 1996) Isolated yields of the ortho-substituted thiazolidine-4-ones 1a-1h are based on aniline as the limiting reactant; no attempt was made to maximize the product yields. Thiazolidin-4-ones were purified by recrystallization using methanol.

3-phenyl-2-(2-trifluoromethylphenyl)-1,3-thiazolidin-4-one (1a). Yield: $(82 \%) ; \mathrm{R}_{\mathrm{f}}=0.463$; m.p. 117-120 ${ }^{\circ} \mathrm{C}$; IR: $\mathrm{cm}^{-1}$ $1683.9(\mathrm{C}=\mathrm{O}) ;{ }^{1} \mathrm{H}$ NMR $\left(\mathrm{CDCl}_{3}\right)$ : 7.65-7.12 $(9 \mathrm{H}$, aromatics), $6.62(1 \mathrm{H}, \mathrm{s}, \mathrm{C} 2), 4.00(1 \mathrm{H}, \mathrm{dd}, \mathrm{C} 5, \mathrm{~J}=1.4 \mathrm{~Hz}$, and $\mathrm{J}=15.8$ Hz), 3.87 (1H, d, C5, J = 15.9 Hz); ${ }^{13}$ C NMR: 171.18 (C4), 139.11, 137.24, 132.82, 129.14, 128.55, 127.34, 126.99, 126.98 $(\mathrm{q}, \mathrm{J}=30.8 \mathrm{~Hz}), 126.06(\mathrm{q}, \mathrm{J}=6.4 \mathrm{~Hz}), 124.92,124.01(\mathrm{q}, \mathrm{J}=274.2 \mathrm{~Hz}), 60.15(\mathrm{~d}, \mathrm{~J}=1.5 \mathrm{~Hz}, \mathrm{C} 2), 33.02(\mathrm{C} 5)$; MS: (m/z) $324\left([\mathrm{M}+\mathrm{H}]^{+}\right), \mathrm{C}_{16} \mathrm{H}_{13} \mathrm{ONSF}_{3}(324.07)$.

2-(2-chlorophenyl)-3-phenyl-1,3-thiazolidin-4-one (1c). Yield (75\%); $\mathrm{R}_{\mathrm{f}}=0.580 ;$ m.p. 90-92 ${ }^{\circ} \mathrm{C}$; IR: $\mathrm{cm}^{-1} 1684.2(\mathrm{C}=\mathrm{O})$, $1053.3(\mathrm{~S}=\mathrm{O}) ;{ }^{1} \mathrm{H}$ NMR $\left(\mathrm{CDCl}_{3}\right): 7.34-7.11(9 \mathrm{H}$, aromatics $), 6.56(1 \mathrm{H}, \mathrm{s}, \mathrm{C} 2), 3.91(1 \mathrm{H}, \mathrm{dd}, \mathrm{C} 5, \mathrm{~J}=1.2 \mathrm{~Hz}$ and $\mathrm{J}=15.8$ $\mathrm{Hz}), 3.78(1 \mathrm{H}, \mathrm{d}, \mathrm{C} 5, \mathrm{~J}=15.7 \mathrm{~Hz}) ;{ }^{13} \mathrm{C}$ NMR: 171.30 (C4), 137.56, 137.06, 132.43, 130.23, 129.72, 129.14, 127.49, 127.09, 126.69, 124.08, 61.63 (C2), 33.01 (C5); MS: (m/z) $290\left([\mathrm{M}+\mathrm{H}]^{+}\right), \mathrm{C}_{15} \mathrm{H}_{13} \mathrm{ONSCl}$ (290.04).

2-(2-fluorophenyl)-3-phenyl-1,3-thiazolidin-4-one (1d). Yield (83\%); $\mathrm{R}_{\mathrm{f}}=0.326 ; \mathrm{m} . \mathrm{p} .137-138{ }^{\circ} \mathrm{C}$; IR: $\mathrm{cm}^{-1} 1667.9$ $(\mathrm{C}=\mathrm{O}), 1055.8(\mathrm{~S}=\mathrm{O}) ;{ }^{1} \mathrm{H}$ NMR $\left(\mathrm{CDCl}_{3}\right): 7.35-6.99(9 \mathrm{H}$, aromatics $), 6.42(1 \mathrm{H}, \mathrm{d}, \mathrm{C} 2, \mathrm{~J}=1.5 \mathrm{~Hz}), 4.03(1 \mathrm{H}, \mathrm{dd}, \mathrm{C} 5, \mathrm{~J}=$ $1.6 \mathrm{~Hz}$ and $\mathrm{J}=17.4 \mathrm{~Hz}), 3.86(1 \mathrm{H}, \mathrm{d}, \mathrm{C} 5, \mathrm{~J}=17.4 \mathrm{~Hz}) ;{ }^{13} \mathrm{C}$ NMR: $171.00(\mathrm{C} 4), 160.26(\mathrm{~d}, \mathrm{~J}=246.7), 137.41,130.59(\mathrm{~d}, \mathrm{~J}=$ $7.8 \mathrm{~Hz}), 129.27,128.12(\mathrm{~d}, \mathrm{~J}=3.0 \mathrm{~Hz}), 127.19,127.03,125.17,124.68(\mathrm{~d}, \mathrm{~J}=4.1 \mathrm{~Hz}), 116.08(\mathrm{~d}, \mathrm{~J}=20.1 \mathrm{~Hz}), 59.35(\mathrm{~d}, \mathrm{C} 2$, $\mathrm{J}=3.1 \mathrm{~Hz}), 33.40(\mathrm{C} 5)$; MS: (m/z) $274\left([\mathrm{M}+\mathrm{H}]^{+}\right), \mathrm{C}_{15} \mathrm{H}_{13} \mathrm{ONSF}(274.33)$.

2-(2-bromophenyl)-3-phenyl-1,3-thiazolidin-4-one (1e). Yield (81\%); $\mathrm{R}_{\mathrm{f}}=0.461 ; \mathrm{m} . \mathrm{p} .90-91{ }^{\circ} \mathrm{C} ; \mathrm{IR}: \mathrm{cm}^{-1} 1683.9(\mathrm{C}=\mathrm{O})$, $1052.8(\mathrm{~S}=\mathrm{O}) ;{ }^{1} \mathrm{H}$ NMR $\left(\mathrm{CDCl}_{3}\right): 7.55-7.11(9 \mathrm{H}$, aromatics $), 6.55(1 \mathrm{H}, \mathrm{s}, \mathrm{C} 2), 3.93(1 \mathrm{H}, \mathrm{d}, \mathrm{C} 5, \mathrm{~J}=15.6 \mathrm{~Hz}), 3.80(1 \mathrm{H}, \mathrm{d}$, 
$\mathrm{C} 5 \mathrm{~J}=15.6 \mathrm{~Hz}$ ); ${ }^{13} \mathrm{C}$ NMR: 171.49 (C4), 138.64 (br), 137.62, 133.53, 130.02, 129.22, 128.20, 126.95 (br), 126.71, 124.01, 122.50, 64.28 (C2), 32.98 (C5); MS: (m/z) $334\left([\mathrm{M}+\mathrm{H}]^{+}\right), \mathrm{C}_{15} \mathrm{H}_{13} \mathrm{ONSBr}(334.24)$.

2-(2-methylphenyl)-3-phenyl-1,3-thiazolidin-4-one (1f). Yield (60\%); $\mathrm{R}_{\mathrm{f}}=0.531 ; \mathrm{m} . \mathrm{p} .80-82{ }^{\circ} \mathrm{C} ; \mathrm{IR}: \mathrm{cm}^{-1} 1682.5(\mathrm{C}=\mathrm{O})$, $1048.4(\mathrm{~S}=\mathrm{O}) ;{ }^{1} \mathrm{H}$ NMR $\left(\mathrm{CDCl}_{3}\right): 7.34-7.11(9 \mathrm{H}$, aromatics), $6.35(1 \mathrm{H}, \mathrm{d}, \mathrm{C} 2, \mathrm{~J}=0.7 \mathrm{~Hz}), 3.94(1 \mathrm{H}, \mathrm{dd}, \mathrm{C} 5 \mathrm{~J}=1.6 \mathrm{~Hz}$ and $\mathrm{J}=16.0 \mathrm{~Hz}), 3.81(1 \mathrm{H}, \mathrm{d}, \mathrm{C} 5, \mathrm{~J}=16.1 \mathrm{~Hz}) ;{ }^{13} \mathrm{C}$ NMR: $171.42(\mathrm{C} 4), 137.93,137.40,134.93,131.19,129.50,129.11$, 126.77, 126.68, 125.71, 124.40, 62.13 (C2), $33.13(\mathrm{C} 5), 19.11\left(\mathrm{CH}_{3}\right)$; MS: (m/z) $286\left([\mathrm{M}+\mathrm{H}]^{+}\right), \mathrm{C}_{16} \mathrm{H}_{16} \mathrm{ONS}(270.37)$.

2-(2-methoxyphenyl)-3-phenyl-1,3-thiazolidin-4-one (1g). Yield (35\%); $\mathrm{R}_{\mathrm{f}}=0.343 ;$ m.p. 82-84 ${ }^{\circ} \mathrm{C}$; IR: $\mathrm{cm}^{-1} 1679.8$ $(\mathrm{C}=\mathrm{O}), 1027.7(\mathrm{~S}=\mathrm{O}) ;{ }^{1} \mathrm{H}$ NMR $\left(\mathrm{CDCl}_{3}\right): 7.34-6.88(9 \mathrm{H}$, aromatics $), 6.46(1 \mathrm{H}, \mathrm{d}, \mathrm{C} 2, \mathrm{~J}=1.0 \mathrm{~Hz}), 3.95(1 \mathrm{H}, \mathrm{dd}, \mathrm{C} 5 \mathrm{~J}=1.4$ $\mathrm{Hz}$ and $\mathrm{J}=15.8 \mathrm{~Hz}), 3.88\left(3 \mathrm{H}, \mathrm{OCH}_{3}\right), 3.78(1 \mathrm{H}, \mathrm{d}, \mathrm{C} 5, \mathrm{~J}=15.5 \mathrm{~Hz}) ;{ }^{13} \mathrm{C}$ NMR: $171.69(\mathrm{C} 4), 156.50,138.05,129.81$, 129.06, 128.02, 126.59, 124.49, 120.83, 111.06, $60.40(\mathrm{C} 2), 55.69\left(\mathrm{OCH}_{3}\right), 33.43(\mathrm{C} 5) ; \mathrm{MS}:(\mathrm{m} / \mathrm{z}) 286\left([\mathrm{M}+\mathrm{H}]^{+}\right)$, $\mathrm{C}_{16} \mathrm{H}_{16} \mathrm{O}_{2} \mathrm{NS}$ (286.37).

2-(2-nitrophenyl)-3-phenyl-1,3-thiazolidin-4-one (1h). Yield (72\%); $\mathrm{R}_{\mathrm{f}}=0.424 ; \mathrm{m} . \mathrm{p} .137-138{ }^{\circ} \mathrm{C} ; \mathrm{IR}: \mathrm{cm}^{-1} 1683.8(\mathrm{C}=\mathrm{O})$, $1062.4(\mathrm{br}, \mathrm{S}=\mathrm{O}) ;{ }^{1} \mathrm{H}$ NMR $\left(\mathrm{CDCl}_{3}\right): 8.12-7.17(9 \mathrm{H}$, aromatics $), 6.81(1 \mathrm{H}, \mathrm{d}, \mathrm{C} 2, \mathrm{~J}=1.0 \mathrm{~Hz}), 3.89(1 \mathrm{H}, \mathrm{dd}, \mathrm{J}=1.2 \mathrm{~Hz}$ and $\mathrm{J}=16.2 \mathrm{~Hz}), 3.77(1 \mathrm{H}, \mathrm{d}, \mathrm{C} 5, \mathrm{~J}=16.2 \mathrm{~Hz}) ;{ }^{13} \mathrm{C}$ NMR: $171.91(\mathrm{C} 4), 146.86,137.66,136.92,134.65,129.58,129.52,127.12$, 126.83, 126.26, 124.11, 60.99 (C2), 32.70 (C5); MS: (m/z) $301\left([\mathrm{M}+\mathrm{H}]^{+}\right), \mathrm{C}_{15} \mathrm{H}_{13} \mathrm{O}_{3} \mathrm{~N}_{2} \mathrm{~S}$ (317.34).

\subsection{General Procedure for the RT Synthesis of Thiazolidin-4-one Sulfoxides via Oxone ${ }^{\circledR}$}

Thiazolidin-4-one $(1.01 \mathrm{mmol})$ was typically dissolved in methanol $(8.0 \mathrm{~mL})$, to which an aqueous solution of Oxone ${ }^{\circledR}$ (461 $\mathrm{mg}, 3.03 \mathrm{mmol}$ calculated as $\mathrm{KHSO}_{5}, 152.2 \mathrm{~g} \mathrm{~mol}^{-1}$, in $4.0 \mathrm{~mL}$ water) was added dropwise at room temperature with vigorous stirring. After the addition, the reaction mixture was stirred for $1 \mathrm{~h}$. Complete conversion of the thiazolidin-4-one was established by thin layer chromatography. Water $(40 \mathrm{~mL})$ was then added to the mixture to dissolve precipitates, and the mixture was extracted with $\mathrm{CHCl}_{3}\left(3 \times 15 \mathrm{~mL}\right.$ ). The combined $\mathrm{CHCl}_{3}$ layers were dried with $\mathrm{Na}_{2} \mathrm{SO}_{4}$, and the $\mathrm{CHCl}_{3}$ was removed in vacuo followed by chromatography. All product sulfoxides with the exception of $\mathbf{5 d}$ were isolated as a mixture of two diastereomeric isomers which were evident by ${ }^{1} \mathrm{H} \mathrm{NMR}$, and ratios of the two isomers were calculated by integration of the hydrogens on $\mathrm{C} 2$.

Sulfoxide-3-phenyl-2-(2-trifluoromethylphenyl)-1,3-thiazolidin-4-one (2a). Yield: (98\%); $\mathrm{R}_{\mathrm{f}}=0.199 ; \mathrm{IR} \mathrm{cm}^{-1} 1696.0$ $(\mathrm{C}=\mathrm{O}), 1053.3(\mathrm{~S}=\mathrm{O}) ;{ }^{1} \mathrm{H}$ NMR $\left(\mathrm{CDCl}_{3}\right), 7.81-7.05(9 \mathrm{H}$, aromatics $)$. Major isomer $(75 \%), 6.22(1 \mathrm{H}, \mathrm{s}, \mathrm{C} 2), 4.01(1 \mathrm{H}, \mathrm{d}$, $\mathrm{C} 5, \mathrm{~J}=17.3 \mathrm{~Hz}), 3.63(1 \mathrm{H}, \mathrm{dd}, \mathrm{C} 5, \mathrm{~J}=0.4 \mathrm{~Hz}$ and $\mathrm{J}=17.4 \mathrm{~Hz})$. Minor isomer $(25 \%), 5.23(1 \mathrm{H}, \mathrm{s}, \mathrm{C} 2), 4.08(1 \mathrm{H}, \mathrm{d}, \mathrm{C} 5, \mathrm{~J}=$ $16.9 \mathrm{~Hz}$ ), $3.78\left(1 \mathrm{H}, \mathrm{d}, \mathrm{C} 5 \mathrm{~J}=17.3 \mathrm{~Hz}\right.$ ); ${ }^{13} \mathrm{C}$ NMR: 168.66 (major C4), 168.40 (minor C4), 137.41, 136.62, 133.28, 131.96, 131.67, 130.24, 129.40, $129.14(\mathrm{q}, \mathrm{J}=26.3 \mathrm{~Hz}), 128.06(\mathrm{q}, \mathrm{J}=33.3 \mathrm{~Hz}), 127.77(\mathrm{q}, \mathrm{J}=5.4 \mathrm{~Hz}), 127.21,126.88,126.80$, $126.45,126.06(\mathrm{q}, \mathrm{J}=5.3 \mathrm{~Hz}), 124.16\left(\mathrm{q}\right.$, minor $\left.\mathrm{CF}_{3}, \mathrm{~J}=274.2 \mathrm{~Hz}\right), 123.86\left(\mathrm{q}\right.$, major $\left.\mathrm{CF}_{3}, \mathrm{~J}=275.5 \mathrm{~Hz}\right), 123.55(\mathrm{q}, \mathrm{J}=$ $273.5 \mathrm{~Hz}$ ), 123.42 (q, J = 3.3 Hz), 123.55, 85.85 (major C2), 75.41 (minor C2), 54.75 (minor C5), 52.19 (major C5); MS: $(\mathrm{m} / \mathrm{z}) 340\left([\mathrm{M}+\mathrm{H}]^{+}\right), \mathrm{C}_{16} \mathrm{H}_{13} \mathrm{O}_{2} \mathrm{NSF}_{3}(340.06)$.

Sulfoxide-2,3-diphenyl-1,3-thiazolidin-4-one (2b). Yield (96\%); $\mathrm{R}_{\mathrm{f}}=0.159 ;$ m.p. $163-164{ }^{\circ} \mathrm{C}: \mathrm{IR}: \mathrm{cm}^{-1} 1681.8(\mathrm{C}=\mathrm{O})$, $1050.5(\mathrm{~S}=\mathrm{O}) ;{ }^{1} \mathrm{H}$ NMR $\left(\mathrm{CDCl}_{3}\right): 7.50-7.21(10 \mathrm{H}$, aromatics). Major isomer $(98 \%), 5.97(1 \mathrm{H}, \mathrm{s}, \mathrm{C} 2), 3.90(1 \mathrm{H}, \mathrm{d}, \mathrm{C} 5, \mathrm{~J}=$ $17.0 \mathrm{~Hz}), 3.58(1 \mathrm{H}, \mathrm{dd}, \mathrm{C} 5 \mathrm{~J}=1.2 \mathrm{~Hz}$ and $\mathrm{J}=16.9 \mathrm{~Hz})$. Minor isomer $(2 \%), 6.07(1 \mathrm{H}, \mathrm{s}, \mathrm{C} 2), 3.98(1 \mathrm{H}, \mathrm{d}, \mathrm{C} 5, \mathrm{~J}=16.6 \mathrm{~Hz})$, $3.79(1 \mathrm{H}, \mathrm{d}, \mathrm{C} 5 \mathrm{~J}=16.6 \mathrm{~Hz}) ;{ }^{13} \mathrm{C}$ NMR: $168.41(\mathrm{C} 4), 138.04,130.73,130.03,129.92,129.36,127.01,126.38,123.50$, 86.41 (C2), 52.28 (C5); MS: (m/z) $272\left([\mathrm{M}+\mathrm{H}]^{+}\right), \mathrm{C}_{15} \mathrm{H}_{14} \mathrm{O}_{2} \mathrm{NS}$ (272.07).

Sulfoxide-2-(2-chlorophenyl)-3-phenyl-1,3-thiazolidin-4-one (2c). Yield (99\%); $\mathrm{R}_{\mathrm{f}}=0.158$; m.p. 60-62 ${ }^{\circ} \mathrm{C}$; IR: $\mathrm{cm}^{-1}$ $1699.6(\mathrm{C}=\mathrm{O}), 1044.5(\mathrm{~S}=\mathrm{O}) ;{ }^{1} \mathrm{H}$ NMR $\left(\mathrm{CDCl}_{3}\right): 7.58-7.15(9 \mathrm{H}$, aromatics). Major isomer $(93 \%), 6.29(1 \mathrm{H}, \mathrm{s}, \mathrm{C} 2), 3.85$ $(1 \mathrm{H}, \mathrm{d}, \mathrm{C} 5, \mathrm{~J}=16.9 \mathrm{~Hz}), 3.64(1 \mathrm{H}, \mathrm{dd}, \mathrm{C} 5 \mathrm{~J}=1.3 \mathrm{~Hz}$ and $\mathrm{J}=17.1 \mathrm{~Hz})$. Minor isomer $(6 \%), 5.32(1 \mathrm{H}, \mathrm{s}, \mathrm{C} 2), 4.11(1 \mathrm{H}, \mathrm{d}$, $\mathrm{C} 5, \mathrm{~J}=16.9 \mathrm{~Hz}), 3.86(1 \mathrm{H}, \mathrm{d}, \mathrm{C} 5 \mathrm{~J}=17.0 \mathrm{~Hz}) ;{ }^{13} \mathrm{C}$ NMR: $168.96(\mathrm{C} 4), 137.87,133.72,131.42,131.15,131.04,129.86$, 129.53, 129.18, 128.32, 128.26, 127.22, 127.11, 126.96, 126.64, 124.41, 123.40, 84.32 (C2), 54.32 (C5 minor), 52.60 (C5 major); MS: (m/z) $306\left([\mathrm{M}+\mathrm{H}]^{+}\right), \mathrm{C}_{15} \mathrm{H}_{13} \mathrm{O}_{2} \mathrm{NSCl}(306.04)$.

Sulfoxide-2-(2-fluorophenyl)-3-phenyl-1,3-thiazolidin-4-one (2d). Yield (99\%); $R_{\mathrm{f}}=0.098$; m.p. 179-180 ${ }^{\circ} \mathrm{C}$; IR: $\mathrm{cm}^{-1}$ $1682.9(\mathrm{C}=\mathrm{O}), 1052.8(\mathrm{~S}=\mathrm{O}) ;{ }^{1} \mathrm{H}$ NMR $\left(\mathrm{CDCl}_{3}\right)$ : $7.48-6.20(9 \mathrm{H}$, aromatics). Major isomer $(89 \%), 6.15(1 \mathrm{H}, \mathrm{s}, \mathrm{C} 2), 3.91$ $(1 \mathrm{H}, \mathrm{d}, \mathrm{C} 5, \mathrm{~J}=17.0 \mathrm{~Hz}), 3.63(1 \mathrm{H}, \mathrm{dd}, \mathrm{C} 5 \mathrm{~J}=0.5 \mathrm{~Hz}$ and $\mathrm{J}=17.1 \mathrm{~Hz})$. Minor isomer $(11 \%), 5.29(1 \mathrm{H}, \mathrm{s}, \mathrm{C} 2), 4.06(1 \mathrm{H}, \mathrm{d}$, $\mathrm{C} 5, \mathrm{~J}=16.7 \mathrm{~Hz}), 3.81(1 \mathrm{H}, \mathrm{d}, \mathrm{C} 5, \mathrm{~J}=16.9 \mathrm{~Hz}) ;{ }^{13} \mathrm{C}$ NMR: $168.54(\mathrm{C} 4), 160.17(\mathrm{~d}, \mathrm{~J}=249.0 \mathrm{~Hz}), 137.70,132.16(\mathrm{~d}, \mathrm{~J}=8.6$ $\mathrm{Hz}), 130.50,129.81,129.52,129.20,127.34,127.11,126.89(\mathrm{~d}, \mathrm{~J}=1.5 \mathrm{~Hz}), 125.54(\mathrm{~d}, \mathrm{~J}=2.9), 124.59,123.82,115.72(\mathrm{~d}, \mathrm{~J}$ $=19.8 \mathrm{~Hz}), 81.58(\mathrm{C} 2), 52.91(\mathrm{C} 5)$; MS: (m/z) $290\left([\mathrm{M}+\mathrm{H}]^{+}\right), \mathrm{C}_{15} \mathrm{H}_{13} \mathrm{O}_{2} \mathrm{NSF}(290.07)$.

Sulfoxide-2-(2-bromophenyl)-3-phenyl-1,3-thiazolidin-4-one (2e). Yield (83\%); $\mathrm{R}_{\mathrm{f}}=0.135$; m.p. 154-155 ${ }^{\circ} \mathrm{C}$; IR: $\mathrm{cm}^{-1}$ $1699.5(\mathrm{C}=\mathrm{O}), 1044.1(\mathrm{~S}=\mathrm{O}) ;{ }^{1} \mathrm{H}$ NMR $\left(\mathrm{CDCl}_{3}\right): 7.65-7.14(9 \mathrm{H}$, aromatics). Major isomer $(82 \%), 6.16(1 \mathrm{H}, \mathrm{s}, \mathrm{C} 2), 3.78$ $(1 \mathrm{H}, \mathrm{d}, \mathrm{C} 5, \mathrm{~J}=17.8 \mathrm{~Hz}), 3.54(1 \mathrm{H}, \mathrm{dd}, \mathrm{C} 5, \mathrm{~J}=1.0 \mathrm{~Hz}$ and $\mathrm{J}=17.2 \mathrm{~Hz})$. Minor isomer $(18 \%), 5.20(1 \mathrm{H}, \mathrm{s}, \mathrm{C} 2), 4.06(1 \mathrm{H}, \mathrm{d}$, 
$\mathrm{C} 5, \mathrm{~J}=16.8 \mathrm{~Hz}), 3.73(1 \mathrm{H}, \mathrm{d}, \mathrm{C} 5, \mathrm{~J}=17.3 \mathrm{~Hz}) ;{ }^{13} \mathrm{C}$ NMR: $169.14(\mathrm{C} 4), 138.00,134.48,133.28,131.68,131.52,129.91$, $129.62,129.29,128.91,127.80,127.27,127.04,126.95,124.46,123.92,123.37,86.57$ (C2 major), 78.70 (C2 minor), 54.43 (C5 minor), 52.43 (C5 major); MS: (m/z) $350\left([\mathrm{M}+\mathrm{H}]^{+}\right), \mathrm{C}_{15} \mathrm{H}_{13} \mathrm{O}_{2} \mathrm{NSBr}(349.99)$.

Sulfoxide-2-(2-methylphenyl)-3-phenyl-1,3-thiazolidin-4-one (2f). Yield (99\%); $R_{\mathrm{f}}=0.154$; m.p. 172-174 ${ }^{\circ} \mathrm{C}$; $\mathrm{IR}: \mathrm{cm}^{-1}$ 1694.2 $(\mathrm{C}=\mathrm{O}), 1055.2(\mathrm{~S}=\mathrm{O}) ;{ }^{1} \mathrm{H}$ NMR $\left(\mathrm{CDCl}_{3}\right)$ : $7.44-7.22(9 \mathrm{H}$, aromatics). Major isomer $(94 \%), 6.07(1 \mathrm{H}, \mathrm{s}, \mathrm{C} 2), 3.90$ $(1 \mathrm{H}, \mathrm{d}, \mathrm{C} 5, \mathrm{~J}=16.9 \mathrm{~Hz}), 3.59(1 \mathrm{H}, \mathrm{dd}, \mathrm{C} 5, \mathrm{~J}=1.1 \mathrm{~Hz}$ and $\mathrm{J}=17.2 \mathrm{~Hz}), 2.60\left(3 \mathrm{H}, \mathrm{s}, \mathrm{CH}_{3}\right)$. Minor isomer $(6 \%), 5.29(1 \mathrm{H}, \mathrm{s}$, $\mathrm{C} 2), 4.03(1 \mathrm{H}, \mathrm{d}, \mathrm{C} 5, \mathrm{~J}=16.1 \mathrm{~Hz}), 3.81(1 \mathrm{H}, \mathrm{d}, \mathrm{C} 5, \mathrm{~J}=16.4 \mathrm{~Hz}), 2.49\left(3 \mathrm{H}, \mathrm{s}, \mathrm{CH}_{3}\right) ;{ }^{13} \mathrm{C}^{\mathrm{NMR}} 168.72(\mathrm{C} 4$ major$), 167.27$ (C4 minor), 138.18, 136.36, 131.98, 131.02, 130.04, 129.76, 129.70, 129.40, 129.09, 128.91, 128.44, 127.48, 127.01, 126.86, 126.49, 126.31, 124.77, 124.28, 123.36, 84.64 (C2), 53.47 (C5 minor), 52.45 (C5 major), $19.46\left(\mathrm{CH}_{3}\right) ; \mathrm{MS}:(\mathrm{m} / \mathrm{z})$ $286\left([\mathrm{M}+\mathrm{H}]^{+}\right), \mathrm{C}_{16} \mathrm{H}_{16} \mathrm{O}_{2} \mathrm{NS}(286.36)$.

Sulfoxide-2-(2-methoxyphenyl)-3-phenyl-1,3-thiazolidin-4-one (2g). Yield (99\%); $\mathrm{R}_{\mathrm{f}}=0.084 ; \mathrm{m} . \mathrm{p} .184-185{ }^{\circ} \mathrm{C} ; \mathrm{IR}_{\mathrm{:}} \mathrm{cm}^{-1}$ $1700.0(\mathrm{C}=\mathrm{O}), 1059.0(\mathrm{~S}=\mathrm{O}) ;{ }^{1} \mathrm{H}$ NMR $\left(\mathrm{CDCl}_{3}\right): 7.50-7.03(9 \mathrm{H}$, aromatics). Major isomer $(85 \%), 6.23(1 \mathrm{H}, \mathrm{s}, \mathrm{C} 2), 4.03$ $\left(3 \mathrm{H}, \mathrm{s}, \mathrm{OCH}_{3}\right), 3.87(1 \mathrm{H}, \mathrm{d}, \mathrm{C} 5, \mathrm{~J}=17.2 \mathrm{~Hz}), 3.60(1 \mathrm{H}, \mathrm{d}, \mathrm{C} 5, \mathrm{~J}=17.0 \mathrm{~Hz})$. Minor isomer $(15 \%), 5.34(1 \mathrm{H}, \mathrm{s}, \mathrm{C} 2), 3.97$ and $3.82\left(4 \mathrm{H}, \mathrm{OCH}_{3}\right.$ and C5) $3.54(1 \mathrm{H}, \mathrm{d}, \mathrm{C} 5, \mathrm{~J}=18.6 \mathrm{~Hz}) ;{ }^{13} \mathrm{C}$ NMR: $169.02(\mathrm{C} 4), 156.84,138.18,131.53,131.22,129.61$, $129.40,129.12,126.99,126.15,124.35,123.29,121.53,120.96,119.90,118.79,111.32,110.83,82.76(\mathrm{C} 2), 55.95\left(\mathrm{OCH}_{3}\right)$, 53.83 (C5 minor), 53.05 (C5 major); MS: (m/z) $302\left([\mathrm{M}+\mathrm{H}]^{+}\right), \mathrm{C}_{16} \mathrm{H}_{16} \mathrm{O}_{3} \mathrm{NS}$ (302.37).

Sulfoxide-2-(2-nitrophenyl)-3-phenyl-1,3-thiazolidin-4-one (2h). Yield (98\%); $\mathrm{R}_{\mathrm{f}}=0.167$; m.p. 78-79 ${ }^{\circ} \mathrm{C}$; IR: $\mathrm{cm}^{-1}$ $1696.8(\mathrm{C}=\mathrm{O}), 1055.8(\mathrm{br}, \mathrm{S}=\mathrm{O}) ;{ }^{1} \mathrm{H}$ NMR $\left(\mathrm{CDCl}_{3}\right): 8.40-7.21(9 \mathrm{H}$, aromatics). Major isomer $(71 \%), 6.68(1 \mathrm{H}, \mathrm{s}, \mathrm{C} 2)$, $3.84(1 \mathrm{H}, \mathrm{d}, \mathrm{C} 5, \mathrm{~J}=17.2 \mathrm{~Hz}), 3.68(1 \mathrm{H}, \mathrm{d}, \mathrm{J}=16.9 \mathrm{~Hz})$. Minor isomer $(29 \%), 5.32(1 \mathrm{H}, \mathrm{s}, \mathrm{C} 2), 4.13(1 \mathrm{H}, \mathrm{d}, \mathrm{C} 5, \mathrm{~J}=16.5$ $\mathrm{Hz}), 3.65(1 \mathrm{H}, \mathrm{d}, \mathrm{J}=16.6 \mathrm{~Hz}) ;{ }^{13} \mathrm{C}$ NMR: 168.73 (C4 major), 166.74 (C4 minor), 148.83, 146.98, 137.64, 137.00, 135.57, 134.05, 131.38, 130.80, 130.38, 129.68, 129.49, 127.56, 127.28, 125.90, 124.80, 124.01, 123.88, 83.96 (C2 major), 75.26 (C2 minor), 53.45 (C5 minor), 52.65 (C5 major); MS: (m/z) 317 ([M+H] $\left.]^{+}\right), \mathrm{C}_{15} \mathrm{H}_{13} \mathrm{O}_{4} \mathrm{~N}_{2} \mathrm{~S}$ (317.33).

Sulfoxide-2-(4-fluorophenyl)-3-phenyl-1,3-thiazolidin-4-one (5a). Yield (97\%); $R_{f}=0.160 ;$ m.p. 192-194 ${ }^{\circ} \mathrm{C} ; I$ : $\mathrm{cm}^{-1}$ 1697.6 $(\mathrm{C}=\mathrm{O}), 1048.3(\mathrm{~S}=\mathrm{O}) ;{ }^{1} \mathrm{H}$ NMR $\left(\mathrm{CDCl}_{3}\right): 7.38-6.98(9 \mathrm{H}$, aromatics). Major isomer $(95 \%), 5.93(1 \mathrm{H}, \mathrm{s}, \mathrm{C} 2), 3.86$ $(1 \mathrm{H}, \mathrm{d}, \mathrm{C} 5, \mathrm{~J}=17.1 \mathrm{~Hz}), 3.56(1 \mathrm{H}, \mathrm{dd}, \mathrm{C} 5, \mathrm{~J}=1.2 \mathrm{~Hz}$ and $\mathrm{J}=17.2 \mathrm{~Hz})$. Minor isomer $(5 \%), 5.25(1 \mathrm{H}, \mathrm{s}, \mathrm{C} 2), 3.96(1 \mathrm{H}, \mathrm{d}$, $\mathrm{C} 5, \mathrm{~J}=16.8 \mathrm{~Hz}$ ), $3.76(1 \mathrm{H}, \mathrm{d}, \mathrm{C} 5, \mathrm{~J}=16.9 \mathrm{~Hz}) ;{ }^{13} \mathrm{C}$ NMR: 168.33 (C4 major), 167.66 (C4 minor), 163.72 (minor, $\mathrm{d}, \mathrm{J}=$ $250.6 \mathrm{~Hz}$ ), 163.49 (major, d, J = 250.6 Hz), 137.90, 136.75, 131.04 (d, J = 7.8 Hz), 129.55, 129.19, $128.43(\mathrm{~d}, \mathrm{~J}=7.7 \mathrm{~Hz})$, 127.28, 127.14, $126.71(\mathrm{~d}, \mathrm{~J}=2.1), 124.97,123.60,117.24(\mathrm{~d}, \mathrm{~J}=22.2 \mathrm{~Hz}), 116.22(\mathrm{~d}, \mathrm{~J}=22.3 \mathrm{~Hz}), 85.84(\mathrm{C} 2), 53.82(\mathrm{C} 5$ minor), 52.91 (C5 major); MS: (m/z) $290\left([\mathrm{M}+\mathrm{H}]^{+}\right), \mathrm{C}_{15} \mathrm{H}_{13} \mathrm{O}_{2} \mathrm{NSF}$ (290.07).

Sulfoxide-2-(4-bromophenyl)-3-phenyl-1,3-thiazolidin-4-one (5b). Yield (98\%); $\mathrm{R}_{\mathrm{f}}=0.183$; m.p. 67-68 ${ }^{\circ} \mathrm{C}$; IR: $\mathrm{cm}^{-1}$ $1698.4(\mathrm{C}=\mathrm{O}), 1050.2(\mathrm{~S}=\mathrm{O}) ;{ }^{1} \mathrm{H} \mathrm{NMR}\left(\mathrm{CDCl}_{3}\right)$ : $7.58-7.21(9 \mathrm{H}$, aromatics). Major isomer $(98 \%), 6.07(1 \mathrm{H}, \mathrm{s}, \mathrm{C} 2), 3.94$ $(1 \mathrm{H}, \mathrm{d}, \mathrm{C} 5, \mathrm{~J}=17.4 \mathrm{~Hz}), 3.64(1 \mathrm{H}, \mathrm{dd}, \mathrm{C} 5, \mathrm{~J}=0.9 \mathrm{~Hz}$ and $\mathrm{J}=17.0 \mathrm{~Hz})$. Minor isomer $(2 \%), 5.29(1 \mathrm{H}, \mathrm{s}, \mathrm{C} 2), 4.07(1 \mathrm{H}, \mathrm{d}$, $\mathrm{C} 5, \mathrm{~J}=16.9 \mathrm{~Hz}), 3.79(1 \mathrm{H}, \mathrm{d}, \mathrm{C} 5 \mathrm{~J}=16.6 \mathrm{~Hz}) ;{ }^{13} \mathrm{C}$ NMR: Major isomer, $168.23(\mathrm{C} 4), 137.57,132.76,129.70,129.19$, 127.98, 126.87, 123.85, 123.27, 85.29 (C2), 52.01 (C5). Minor isomer, 167.95 (C4), 136.51, 131.71, 130.55, 128.81, 127.64, 126.69, 124.78, 124.07, 85.29 (C2), 53.87 (C5); MS: (m/z) $350\left([\mathrm{M}+\mathrm{H}]^{+}\right), \mathrm{C}_{15} \mathrm{H}_{13} \mathrm{O}_{2} \mathrm{NSBr}$ (349.99).

Sulfoxide-2-(4-methoxyphenyl)-3-phenyl-1,3-thiazolidin-4-one (5c). Yield (99\%); $\mathrm{R}_{\mathrm{f}}=0.103$; m.p. 156-157 ${ }^{\circ} \mathrm{C}$; IR: $\mathrm{cm}^{-1}$ $1705.2(\mathrm{C}=\mathrm{O}), 1051.9(\mathrm{~S}=\mathrm{O}) ;{ }^{1} \mathrm{H}$ NMR $\left(\mathrm{CDCl}_{3}\right): 7.42-6.82(9 \mathrm{H}$, aromatics). Major isomer $(86 \%), 5.94(1 \mathrm{H}, \mathrm{s}, \mathrm{C} 2), 3.90$ $(1 \mathrm{H}, \mathrm{d}, \mathrm{C} 5, \mathrm{~J}=17.4 \mathrm{~Hz}), 3.78\left(3 \mathrm{H}, \mathrm{s}, \mathrm{OCH}_{3}\right), 3.54(1 \mathrm{H}, \mathrm{dd}, \mathrm{C} 5, \mathrm{~J}=1.2 \mathrm{~Hz}$ and $\mathrm{J}=17.0 \mathrm{~Hz})$. Minor isomer $(14 \%), 5.26(1 \mathrm{H}$, s, C2), $3.96(1 \mathrm{H}, \mathrm{d}, \mathrm{C} 5, \mathrm{~J}=17.0 \mathrm{~Hz}), 3.78$ and $3.71\left(4 \mathrm{H}, \mathrm{OCH}_{3}\right.$ and $\left.\mathrm{C} 5\right) ;{ }^{13} \mathrm{C}$ NMR: Major isomer, $168.34(\mathrm{C} 4), 160.71$, $138.04,129.28,127.73,126.90,123.43,122.23,115.25,85.95(\mathrm{C} 2), 55.45\left(\mathrm{OCH}_{3}\right), 52.17(\mathrm{C} 5)$. Minor isomer, $167.71(\mathrm{C} 4)$, 136.95, 130.30, 128.91, 126.76, 124.89, 120.00, 114.28, $85.95(\mathrm{C} 2), 55.20\left(\mathrm{OCH}_{3}\right), 53.65(\mathrm{C} 5) ; \mathrm{MS}:(\mathrm{m} / \mathrm{z}) 302\left([\mathrm{M}+\mathrm{H}]^{+}\right)$, $\mathrm{C}_{16} \mathrm{H}_{16} \mathrm{O}_{3} \mathrm{NS}$ (302.37).

Sulfoxide-2-(2-nitrophenyl)-3-phenyl-1,3-thiazolidin-4-one (5d). Yield (77\%); $\mathrm{R}_{\mathrm{f}}=0.075$; m.p. d $213{ }^{\circ} \mathrm{C}$; IR: $\mathrm{cm}^{-1} 1701.3$ $(\mathrm{C}=\mathrm{O}), 1051.8(\mathrm{~S}=\mathrm{O}) ;{ }^{1} \mathrm{H}$ NMR $\left(\mathrm{CDCl}_{3}\right): 8.35-7.27(9 \mathrm{H}$, aromatics). $6.04(1 \mathrm{H}, \mathrm{s}, \mathrm{C} 2), 3.99(1 \mathrm{H}, \mathrm{d}, \mathrm{C} 5, \mathrm{~J}=17.2 \mathrm{~Hz}), 3.69$ $(1 \mathrm{H}, \mathrm{dd}, \mathrm{J}=0.8 \mathrm{~Hz}$ and $17.4 \mathrm{~Hz}) ;{ }^{13} \mathrm{C}$ NMR: 168.15 (C4), 148.98, 138.14, 137.58, 129.86, 127.76, 127.67, 125.32, 123.74, 86.12 (C2), 52.62 (C5); MS: (m/z) $317\left([\mathrm{M}+\mathrm{H}]^{+}\right), \mathrm{C}_{15} \mathrm{H}_{13} \mathrm{O}_{4} \mathrm{~N}_{2} \mathrm{~S}$ (317.33).

\subsection{General Procedure for the Synthesis of Thiazolidin-4-One Sulfones via $\mathrm{KMnO}_{4}$}

Thiazolidin-4-one $(0.553 \mathrm{mmol})$ was dissolved in glacial acetic acid $(2.4 \mathrm{~mL})$, to which an aqueous solution of $\mathrm{KMnO}_{4}(175$ $\mathrm{mg}, 1.11 \mathrm{mmol}$, in $3.0 \mathrm{~mL}$ water) was added dropwise at room temperature with vigorous stirring, and stirred an additional 5 $\mathrm{m}$. Solid sodium bisulfite $\left(\mathrm{NaHSO}_{3} / \mathrm{Na}_{2} \mathrm{~S}_{2} \mathrm{O}_{5}\right.$ ) was then added until the solution remained colorless; $3.0 \mathrm{~mL}$ of water was then added to the mixture and stirred for $10 \mathrm{~m}$. Most crude products were isolated as powders by filtration and water rinses; products were purified by recrystallization in $\mathrm{CH}_{3} \mathrm{OH}$. Products $3 \mathbf{a}, \mathbf{3 c}$, and $\mathbf{3 e}$ were not isolated as powders, but rather by 
extraction of the reaction mixture with toluene $(3 \times 10 \mathrm{~mL})$. The combined toluene layers were dried with $\mathrm{Na}_{2} \mathrm{SO}_{4}$, and toluene was removed in vacuo followed by chromatography.

Sulfone-3-phenyl-2-(2-trifluoromethylphenyl)-1,3-thiazolidin-4-one (3a). Yield: (73\%); oil; $\mathrm{R}_{\mathrm{f}}=0.314$; $\mathrm{IR} \mathrm{cm}^{-1} 1705.2$ $(\mathrm{C}=\mathrm{O}), 1128.2,1120.4(\mathrm{~S}=\mathrm{O}) ;{ }^{1} \mathrm{H}$ NMR $\left(\mathrm{CDCl}_{3}\right), 7.76-7.20(9 \mathrm{H}$, aromatics $), 6.52(1 \mathrm{H}, \mathrm{s}, \mathrm{C} 2), 4.15(1 \mathrm{H}, \mathrm{d}, \mathrm{C} 5, \mathrm{~J}=16.8$ $\mathrm{Hz}), 4.09(1 \mathrm{H}, \mathrm{d}, \mathrm{C} 5 \mathrm{~J}=16.8 \mathrm{~Hz}) ;{ }^{13} \mathrm{C}$ NMR: $162.45(\mathrm{C} 4), 135.66,132.86,130.72,129.91(\mathrm{q}, \mathrm{J}=31.1 \mathrm{~Hz}), 129.58,128.45$, 128.19, 127.85, 127.21 (br q, J = 6.0 Hz), 125.25, 123.67 (q, J = $274.3 \mathrm{~Hz}), 80.01$ (C2), 50.97 (C5); MS: (m/z) 356 $\left([\mathrm{M}+\mathrm{H}]^{+}\right), \mathrm{C}_{16} \mathrm{H}_{13} \mathrm{O}_{3} \mathrm{NSF}_{3}(356.06)$.

Sulfone-2,3-diphenyl-1,3-thiazolidin-4-one (3b). Yield (76\%); $R_{\mathrm{f}}=0.366 ;$ m.p. $178-180{ }^{\circ} \mathrm{C}: \mathrm{IR}: \mathrm{cm}^{-1} 1693.1(\mathrm{C}=\mathrm{O})$, 1141.6, $1125.2(\mathrm{~S}=\mathrm{O}) ;{ }^{1} \mathrm{H}$ NMR $\left(\mathrm{CDCl}_{3}\right): 7.47-7.18(10 \mathrm{H}$, aromatics $), 5.99(1 \mathrm{H}, \mathrm{s}, \mathrm{C} 2), 3.88(1 \mathrm{H}, \mathrm{d}, \mathrm{C} 5, \mathrm{~J}=16.9 \mathrm{~Hz})$, $3.56(1 \mathrm{H}, \mathrm{dd}, \mathrm{C} 5, \mathrm{~J}=1.2 \mathrm{~Hz}$ and $\mathrm{J}=17.1 \mathrm{~Hz}) ;{ }^{13} \mathrm{C}$ NMR: $162.58(\mathrm{C} 4), 136.25,130.87,129.62,129.58,129.01,128.05$, 125.13, 83.92 (C2), 50.70 (C5); MS: (m/z) $288\left([\mathrm{M}+\mathrm{H}]^{+}\right), \mathrm{C}_{15} \mathrm{H}_{14} \mathrm{O}_{3} \mathrm{NS}(288.07)$.

Sulfone-2-(2-chlorophenyl)-3-phenyl-1,3-thiazolidin-4-one (3c). Yield (69\%); oil; $\mathrm{R}_{\mathrm{f}}=0.328$; IR: $\mathrm{cm}^{-1} 1717.6(\mathrm{C}=\mathrm{O})$, 1140.6, $1097.0(\mathrm{~S}=\mathrm{O}) ;{ }^{1} \mathrm{H}$ NMR $\left(\mathrm{CDCl}_{3}\right): 7.53-7.26(9 \mathrm{H}$, aromatics $), 6.65(1 \mathrm{H}, \mathrm{s}, \mathrm{C} 2), 4.10(1 \mathrm{H}, \mathrm{d}, \mathrm{C}, \mathrm{J}=16.9 \mathrm{~Hz}), 4.05$ $(1 \mathrm{H}, \mathrm{d}, \mathrm{C} 5, \mathrm{~J}=16.6 \mathrm{~Hz}) ;{ }^{13} \mathrm{C}$ NMR: 162.50 (C4), 136.12, 135.29 (br), 131.80, 130.93 (br), 129.64, 128.05, 127.85, 127.65, 127.54 (br), 124.74, $80.48(\mathrm{C} 2), 50.78$ (C5); MS: (m/z) $322\left([\mathrm{M}+\mathrm{H}]^{+}\right), \mathrm{C}_{15} \mathrm{H}_{13} \mathrm{O}_{3} \mathrm{NSCl}(322.03)$.

Sulfone-2-(2-fluorophenyl)-3-phenyl-1,3-thiazolidin-4-one (3d). Yield (76\%); m.p. 159-160 ${ }^{\circ} \mathrm{C}$; IR: $\mathrm{cm}^{-1} 1686.5(\mathrm{C}=\mathrm{O})$, 1136.0, 1127.2 (S=O); ${ }^{1} \mathrm{H}$ NMR $\left(\mathrm{CDCl}_{3}\right): 7.53-7.22(9 \mathrm{H}$, aromatics), $6.27(1 \mathrm{H}, \mathrm{s}, \mathrm{C} 2), 4.17(1 \mathrm{H}, \mathrm{d}, \mathrm{C} 5, \mathrm{~J}=16.9 \mathrm{~Hz}), 4.10$ $(1 \mathrm{H}, \mathrm{d}, \mathrm{C} 5, \mathrm{~J}=17.0 \mathrm{~Hz}) ;{ }^{13} \mathrm{C}$ NMR: $162.40(\mathrm{C} 4), 161.49$ (d, J = 250.9), 135.99, 132.87 (d, J = 7.8 Hz), 129.75, 128.98, 128.38, 125.34, 125.24 (d, J = 3.1 Hz), 117.70 (d, J = 11.7), 116.85 (d, J = 20.8 Hz), 79.78 (C2), 51.26 (C5); MS: (m/z) 306 $\left([\mathrm{M}+\mathrm{H}]^{+}\right), \mathrm{C}_{15} \mathrm{H}_{13} \mathrm{O}_{3} \mathrm{NSF}(306.06)$.

Sulfone-2-(2-bromophenyl)-3-phenyl-1,3-thiazolidin-4-one (3e). Yield (91\%); oil; $\mathrm{R}_{\mathrm{f}}=0 . .270$; IR: $\mathrm{cm}^{-1} 1718.3(\mathrm{C}=\mathrm{O})$, 1141.5, $1097.8(\mathrm{~S}=\mathrm{O}) ;{ }^{1} \mathrm{H}$ NMR $\left(\mathrm{CDCl}_{3}\right): 7.70-7.16(9 \mathrm{H}$, aromatics), $6.68(1 \mathrm{H}, \mathrm{s}, \mathrm{C} 2), 4.09(1 \mathrm{H}, \mathrm{d}, \mathrm{C}, \mathrm{J}=16.9 \mathrm{~Hz}), 4.04$ $(1 \mathrm{H}, \mathrm{d}, \mathrm{C} 5 \mathrm{~J}=16.9 \mathrm{~Hz}) ;{ }^{13} \mathrm{C}$ NMR: $162.44(\mathrm{C} 4), 136.09,134.15,131.98,129.60,129.15,129.01,128.40,127.97,127.82$, 124.68, 83.79 (C2), 50.87 (C5); MS: (m/z) $366\left([\mathrm{M}+\mathrm{H}]^{+}\right), \mathrm{C}_{15} \mathrm{H}_{13} \mathrm{O}_{3} \mathrm{NSBr}(365.98)$.

Sulfone-2-(2-methylphenyl)-3-phenyl-1,3-thiazolidin-4-one (3f). Yield (70\%); m.p. 143-144 ${ }^{\circ} \mathrm{C}$; IR: $\mathrm{cm}^{-1} 1698.5(\mathrm{C}=\mathrm{O})$, 1129.0, $1100.5(\mathrm{~S}=\mathrm{O}) ;{ }^{1} \mathrm{H}$ NMR $\left(\mathrm{CDCl}_{3}\right): 7.39-7.25(9 \mathrm{H}$, aromatics), $6.27(1 \mathrm{H}, \mathrm{s}, \mathrm{C} 2), 4.08(1 \mathrm{H}, \mathrm{d}, \mathrm{C} 5, \mathrm{~J}=16.3 \mathrm{~Hz}), 4.00$ $(1 \mathrm{H}, \mathrm{d}, \mathrm{C} 5, \mathrm{~J}=16.8 \mathrm{~Hz}), 2.50\left(3 \mathrm{H}, \mathrm{s}, \mathrm{CH}_{3}\right) ;{ }^{13} \mathrm{C}$ NMR: $162.73(\mathrm{C} 4), 138.21,136.61,131.98,130.50,129.60,127.96,127.14$, 125.64, 124.71, $81.20(\mathrm{C} 2), 50.56(\mathrm{C} 5), 20.00\left(\mathrm{CH}_{3}\right)$; MS: (m/z) $302\left([\mathrm{M}+\mathrm{H}]^{+}\right), \mathrm{C}_{16} \mathrm{H}_{16} \mathrm{O}_{3} \mathrm{NS}(302.32)$.

Sulfone-2-(2-methoxyphenyl)-3-phenyl-1,3-thiazolidin-4-one (3g). Yield (80\%); m.p. 130-131 ${ }^{\circ} \mathrm{C}$; IR: $\mathrm{cm}^{-1} 1699.6(\mathrm{C}=\mathrm{O})$, 1141.9, $1127.7(\mathrm{~S}=\mathrm{O}) ;{ }^{1} \mathrm{H}$ NMR $\left(\mathrm{CDCl}_{3}\right): 7.46-7.00(9 \mathrm{H}$, aromatics), $6.32(1 \mathrm{H}, \mathrm{vbr} \mathrm{s}, \mathrm{C} 2),, 4.05(1 \mathrm{H}, \mathrm{d}, \mathrm{C} 5, \mathrm{~J}=16.5 \mathrm{~Hz})$, $3.96(1 \mathrm{H}, \mathrm{d}, \mathrm{C} 5, \mathrm{~J}=17.0 \mathrm{~Hz}), 3.93\left(3 \mathrm{H}, \mathrm{s}, \mathrm{OCH}_{3}\right) ;{ }^{13} \mathrm{C}$ NMR: $162.94(\mathrm{C} 4), 158.28,136.59,132.16,129.56,128.00,125.13$ (br), 121.32, 118.62, 111.85, 80.48 (vbr, C2), $56.04\left(\mathrm{OCH}_{3}\right), 51.19$ (br, C5); MS: (m/z) $318\left([\mathrm{M}+\mathrm{H}]^{+}\right), \mathrm{C}_{16} \mathrm{H}_{16} \mathrm{O}_{4} \mathrm{NS}$ (318.36).

Sulfone-2-(2-nitrophenyl)-3-phenyl-1,3-thiazolidin-4-one (3h). Yield (63\%); m.p. 177-179 ${ }^{\circ} \mathrm{C}$; IR: $\mathrm{cm}^{-1} 1709.0(\mathrm{C}=\mathrm{O})$, 1158.8, 1120.2 (S=O); ${ }^{1} \mathrm{H}$ NMR $\left(\mathrm{CDCl}_{3}\right): 8.38-7.27(9 \mathrm{H}$, aromatics), $7.19(1 \mathrm{H}, \mathrm{s}, \mathrm{C} 2), 4.03(1 \mathrm{H}, \mathrm{d}, \mathrm{C} 5, \mathrm{~J}=16.4 \mathrm{~Hz}), 3.94$ $(1 \mathrm{H}, \mathrm{d}, \mathrm{C} 5, \mathrm{~J}=17.1 \mathrm{~Hz}) ;{ }^{13} \mathrm{C}$ NMR: $162.64(\mathrm{C} 4), 148.34,136.48,135.09,131.69,129.91,128.35,127.27,127.22,127.12$, 124.62, 80.98 (C2), 50.69 (C5); MS: (m/z) $333\left([\mathrm{M}+\mathrm{H}]^{+}\right), \mathrm{C}_{15} \mathrm{H}_{13} \mathrm{O}_{5} \mathrm{~N}_{2} \mathrm{~S}$ (333.32).

Sulfone-2-(4-fluorophenyl)-3-phenyl-1,3-thiazolidin-4-one (6a). Yield (81\%); m.p. 186-187 ${ }^{\circ} \mathrm{C}$; IR: $\mathrm{cm}^{-1} 1723.8(\mathrm{C}=\mathrm{O})$, 1189.3, $1095.9(\mathrm{~S}=\mathrm{O}) ;{ }^{1} \mathrm{H}$ NMR $\left(\mathrm{CDCl}_{3}\right)$ : $7.35-7.07\left(9 \mathrm{H}\right.$, aromatics), $5.96(1 \mathrm{H}, \mathrm{s}, \mathrm{C} 2), 4.02(2 \mathrm{H}, \mathrm{s}) ;{ }^{13} \mathrm{C}$ NMR: $163.93(\mathrm{~d}$, $\mathrm{J}=303.1), 162.93(\mathrm{C} 4), 135.93,130.37(\mathrm{~d}, \mathrm{~J}=8.9 \mathrm{~Hz}), 129.68,128.22,125.28,124.41(\mathrm{~d}, \mathrm{~J}=3.1 \mathrm{~Hz}), 116.92(\mathrm{~d}, \mathrm{~J}=22.0$ $\mathrm{Hz}), 83.04$ (C2), 50.88 (C5); MS: (m/z) $306\left([\mathrm{M}+\mathrm{H}]^{+}\right), \mathrm{C}_{15} \mathrm{H}_{13} \mathrm{O}_{3} \mathrm{NSF}(306.06)$.

Sulfone-2-(4-bromophenyl)-3-phenyl-1,3-thiazolidin-4-one (6b). Yield (78\%); m.p. 177-178 ${ }^{\circ} \mathrm{C}$; IR: $\mathrm{cm}^{-1} 1680.6(\mathrm{C}=\mathrm{O})$, 1127.4, $1104.0(\mathrm{~S}=\mathrm{O}) ;{ }^{1} \mathrm{H}$ NMR $\left(\mathrm{CDCl}_{3}\right): 7.61-7.27$ (9 H, aromatics), $6.02(1 \mathrm{H}, \mathrm{s}, \mathrm{C} 2), 4.08(2 \mathrm{H}, \mathrm{s}) ;{ }^{13} \mathrm{C}$ NMR: 162.39 (C4), 135.84, 132.82, 129.81, 129.65, 128.18, 127.66, 125.38, 125.15, 83.07 (C2), 50.84 (C5); MS: (m/z) $366\left([\mathrm{M}+\mathrm{H}]^{+}\right)$, $\mathrm{C}_{15} \mathrm{H}_{13} \mathrm{O}_{3} \mathrm{NSBr}$ (365.98).

Sulfone-2-(4-methoxyphenyl)-3-phenyl-1,3-thiazolidin-4-one (6c). Yield (88\%); m.p. 124-127 ${ }^{\circ} \mathrm{C}$; IR: $\mathrm{cm}^{-1} 1708.2(\mathrm{C}=\mathrm{O})$, 1121.0, 1100.4 (S=O); ${ }^{1} \mathrm{H}$ NMR $\left(\mathrm{CDCl}_{3}\right): 7.35-6.92(9 \mathrm{H}$, aromatics), $5.98(1 \mathrm{H}, \mathrm{s}, \mathrm{C} 2),, 4.07(1 \mathrm{H}, \mathrm{d}, \mathrm{C} 5, \mathrm{~J}=16.2 \mathrm{~Hz}), 4.02$ $(1 \mathrm{H}, \mathrm{d}, \mathrm{C} 5, \mathrm{~J}=16.8 \mathrm{~Hz}), 3.80\left(3 \mathrm{H}, \mathrm{s}, \mathrm{OCH}_{3}\right) ;{ }^{13} \mathrm{C}$ NMR: $162.61(\mathrm{C} 4), 161.42,136.11,129.68,129.46,127.92,125.28$, 120.12, 114.96, $83.34(\mathrm{C} 2), 55.48\left(\mathrm{OCH}_{3}\right), 50.70(\mathrm{C} 5) ; \mathrm{MS}:(\mathrm{m} / \mathrm{z}) 318\left([\mathrm{M}+\mathrm{H}]^{+}\right), \mathrm{C}_{16} \mathrm{H}_{16} \mathrm{O}_{4} \mathrm{NS}(318.36)$.

Sulfone-2-(4-nitrophenyl)-3-phenyl-1,3-thiazolidin-4-one (6d). Yield (82\%); m.p. 204-206 ${ }^{\circ} \mathrm{C}$; IR: $\mathrm{cm}^{-1} 1698.8(\mathrm{C}=\mathrm{O})$, 1130.7, $1113.9(\mathrm{~S}=\mathrm{O})$; ${ }^{1} \mathrm{H}$ NMR $\left(\mathrm{CDCl}_{3}\right): 8.32-7.28(9 \mathrm{H}$, aromatics $), 6.17(1 \mathrm{H}, \mathrm{s}, \mathrm{C} 2), 4.17(1 \mathrm{H}, \mathrm{d}, \mathrm{C} 5, \mathrm{~J}=16.9 \mathrm{~Hz}), 4.11$ 
$(1 \mathrm{H}, \mathrm{d}, \mathrm{C} 5, \mathrm{~J}=16.9 \mathrm{~Hz}) ;{ }^{13} \mathrm{C}$ NMR: $162.06(\mathrm{C} 4), 149.40,135.53,135.39,129.89,129.52,128.50,125.09,124.68,82.69$ (C2), 51.18 (C5); MS: (m/z) $333\left([\mathrm{M}+\mathrm{H}]^{+}\right), \mathrm{C}_{15} \mathrm{H}_{13} \mathrm{O}_{5} \mathrm{~N}_{2} \mathrm{~S}(333.32)$.

\subsection{High Temperature Oxidation of Thiazolidin-4-Ones 1a-h Using Oxone ${ }^{\circledR}$}

Thiazolidin-4-one (1a-h) $(0.103 \mathrm{mmol})$ was dissolved in refluxing $\mathrm{MeOH}(16 \mathrm{~mL})$, to which a solution of Oxone ${ }^{\circledR}(1.251 \mathrm{~g}$, $0.824 \mathrm{mmol}$, in $8 \mathrm{~mL} \mathrm{H}_{2} \mathrm{O}$ ) was added dropwise with vigorous stirring. Then the reaction mixture was heated for $8 \mathrm{~h}$. Upon cooling, $40 \mathrm{~mL}$ of $\mathrm{H}_{2} \mathrm{O}$ was added and the solution was then extracted with $\mathrm{CH}_{2} \mathrm{Cl}_{2}(3 \times 25 \mathrm{~mL})$. The combined $\mathrm{CH}_{2} \mathrm{Cl}_{2}$ layers were dried with $\mathrm{Na}_{2} \mathrm{SO}_{4}$, and the $\mathrm{CH}_{2} \mathrm{Cl}_{2}$ was removed in vacuo. The resulting crude product mixture of sulfoxide and sulfone was purified using preparative chromatography plates (silica gel GF, 250 micron) purchased from Analtech (Rabel \& Sherma, 2017). The product mixture was dissolved sparingly in $\mathrm{CH}_{2} \mathrm{Cl}_{2}$ and deposited on the preparative chromatography plates using a streaking apparatus purchased from the Aldrich Chemical Company. No more than $150 \mathrm{mg}$ of product mixture was deposited on a plate. The plates were developed using 3:1 cyclohexane:ethyl acetate, after which the plates were air-dried. The location of the products' bands on the plate were identified by $\mathrm{R}_{\mathrm{f}}$ values and fluorescent indicators present in the silica gel. The sulfoxide and sulfone bands were separated by scraping the bands of silica from the plate. The products were then isolated by extracting the collected silica with boiling ethyl acetate followed by filtration and removal of the ethyl acetate in vacuo. The molar ratios of either sulfoxide $\mathbf{2}$ to sulfone $\mathbf{3}$ or sulfoxide $\mathbf{5}$ to sulfone $\mathbf{6}$ were determined by the isolated yields of the two respective compounds based on the thiazolidin-4-one $\mathbf{1}$ as the limiting reagent.

\section{Acknowledgements}

We would like to thank Dr. Charles DeBrosse and the Chemistry Department of Temple University for allowing us to run the NMR spectra on their spectrometer.

\section{References}

Abhinit, M., Ghodke, M., \& Pratima, N. A. (2009). Exploring potential of 4-thiazolidinone: a brief review. Int. J. Pharmacy Pharm. Sci., 1, 47-64.

Brown, F. C. (1961). 4-Thiazolidinones. Chem. Rev., 61, 463-521. https://doi.org/10.1021/cr60213a002

Cannon, K. C., Costa, M., Pepper, M., Toovy, J., Selinsky, R. S., \& Lagalante, A. F. (2017). Selective synthesis of meta- and para-substituted 3-aryl-2-phenyl-1,3-thiazolidin-4-one sulfoxides and sulfones by S-oxidation with oxone ${ }^{\circledR}$, Intl. J. Chem., 9(4), in press. https://doi.org/10.5539/ijc.v9n4p1

Cannon, K. C., Gandla, D., Lauro, S., Silverberg, L. J., Tierney, J., \& Lagalante, A. (2015). Selective synthesis of ortho-substituted 3-cyclohexyl-2-phenyl-1,3-thiazolidin-4-one sulfoxides and sulfones by $S$-oxidation with Oxone ${ }^{\circledR}$, Intl. J. Chem., 7(2), 73-83. https://doi.org/10.5539/ijc.v7n2p73

Colombo, A., Fernandez, J. C., Fernandez-Forner, D., de la Figuera, N., Albericio, F., \& Forns, P. (2008). Stereomeric studies on the oxidation and alkylation of 4-thiazolidinones. Tet. Lett., 49, 1569-1572. https://doi.org/10.1016/j.tetlet.2008.01.038

Gududuru, V., Hurh, E., Dalton, J. T., \& Miller, D. D. (2004). Discovery of 2-arylthiazolidine-4-carboxylic acid amides as a new class of cytotoxic agents for prostate cancer. Bioorg. Med. Chem. Lett., 14, 5289-5293. https://doi.org/10.1016/j.bmcl.2004.08.029

Hamama, W. S., Ismail, M. A., Shaaban, S., \& Zoorob, H. H. (2008). Progress in the chemistry of 4-thioazolidinones. J. Het. Chem., 45, 939-956. https://doi.org/10.1002/jhet.5570450401

Hussain, H., Green, I. R., \& Ahmed, I. (2013). Journey describing applications of oxone in synthetic chemistry. Chem. Rev., 113, 3329-3371. https://doi.org/10.1021/cr3004373

Jain, A. K., Vaidya, A., Ravichandran, V., Kashaw, S. K., \& Agrawal, R. A. (2012). Recent developments and biological activities of thiazolidinone derivatives: a review. Bioorg. Med. Chem., 20, 3378-3395. https://doi.org/10.1016/j.bmc.2012.03.069

Kaushal, M., \& Kaur, A. (2016). A review on some 2,5-disubstituted [1,3,4] thiadiazole substituted thiazolidinone derivatives as a potent antimicrobial agents. World Journal of Pharmaceutical Research, 5(6), 1966-1977.

Kumar, D., Kumar, V., Mundlia, J., Pradhan, D., \& Malik, S. (2015). Thiazolidin-4-one Derivatives as Central Nervous System Potential Agents. Central Nervous System Agents in Medicinal Chemistry, 15(1), 23-27. https://doi.org/10.2174/1871524915666150220111509

Lee, J. B., Yergatian, S. Y., Crowther, B. C., \& Downie, I. M. (1990). Preparation of 4-thiazolidinone 1-oxides. Org. Prep. Proc. Int., 22, 544-546. https://doi.org/10.1080/00304949009356324 
Madesclaire, M. (1986). Reduction of sulfoxides to thioethers. Tetrahedron, 42, 5459-5480. https://doi.org/10.1016/S0040-4020(01)88150-3

Omar, M. T., El-Kharmry, A. E., \& Sherif, F. A. (1981). Synthesis of 2,3-disubstituted-4-oxothiazolidine 1-oxides. J. Het. Chem., 18, 633-634. https://doi.org/10.1002/jhet.5570180340

Rabel, F., \& Sherma, J. (2017). Review of the state of the art of preparative thin-layer chromatography. J. Liquid Chromatography and Related Technologies, 40(4), 165-176. https://doi.org/10.1080/10826076.2017.1294081

Rozwadowska, M. D., \& Sulima, A. (2003). Studies of some hydrogenated thiazolo[2,3-a]isoquinoline $S$-oxides. Tetrahedron, 59, 1173-1179. https://doi.org/10.1016/S0040-4020(03)00023-1

Rozwadowska, M. D., Sulima, A., \& Gzellla, A. (2002). Synthesis, crystal structure and oxidation of (R)-(+)-8,9-dimethoxy-6,10b-dihydro-5H-thiazolo[2,3-a]isoquinolin-3-one. $\quad$ Tet. $\quad$ Asymm., $13, \quad$ 2329-2333. https://doi.org/10.1016/S0957-4166(02)00633-X

Singh, S. P., Parmar, S. S., Raman, R., Virgil, I., \& Stenberg, V. I. (1981). Chemistry and biological activity of thiazolidinones. Chem. Rev., 81, 175-203. https://doi.org/10.1021/cr00042a003

Smith, R. L., Lee, T. J., \& Cragoe, E. J. (1977). Certain thiazolidine compounds. U.S. Patent 4059587.

Surrey, A. R. (1948). The preparation of 2,3-disubstituted-4-thiazolidones. II. 3-Alkyl (and aralkyl) 2-aryl derivatives. J. Am. Chem. Soc., 70, 4262-4263. https://doi.org/10.1021/ja01192a515

Surrey, A. R. (1967). 3-[(2-Oxazolidinone-3-yl)-alkyl]-4-thiazolidinones and their preparation. U.S. Patent 3309377.

Suryawanshi, R., Jadhav, S., Makwana, N., Desai, D., Chaturbhuj, D., Sonawani, A., ... Tripathy, S. (2017). Evaluation of 4-thiazolidinone derivatives as potential reverse transcriptase inhibitors against HIV-1 drug resistant strains. Bioorganic Chem., 71, 211-218. https://doi.org/10.1016/j.bioorg.2017.02.007

Tierney, J., Houghton, G., Sanford, K., Mascavage, L., McCoy, M., Findeisen, A., \& Kilburn, J. (1996). Effects and conformational analysis of some substituted 2,3-diphenyl-1,3-thiazolidin-4-ones. Magnetic Resonance Chemistry, 34, 573-576. https://doi.org/10.1002/(SICI)1097-458X(199608)34:8<573::AID-OMR928>3.0.CO;2-D

Tierney, J., Sheridan, D., Mascavage, L., Gorbecheva, D., Ripp, M., \& Son, S. (2005). A preliminary study on predicting ${ }^{13} \mathrm{C}$ chemical shifts for a series of disubstituted 2,3-diphenyl-1,3-thiazolidin-4-ones. Heterocyclic Communications, 11(3-4), 215-222. https://doi.org/10.1515/HC.2005.11.3-4.215

Tripathi, A. C., Gupta, S. J., Fatima, G. N., Sonar, P. K., Verma, A., \& Saraf, S. K. (2014). 4-Thiazolidinones: the advances continue.... Eur. J. Med. Chem., 72, 52-77. https://doi.org/10.1016/j.ejmech.2013.11.017

Trost, B. M., \& Curran, D. P. (1981). Chemoselective oxidation of sulfides to sulfones with potassium hydrogen persulfate. Tet. Lett., 22, 1287-1290. https://doi.org/10.1016/S0040-4039(01)90298-9

Troutman, H. D., \& Long, L. M. (1948). The synthesis of 2,3-disubstituted-4-thiazolidones. J. Am. Chem. Soc., 70, 3436-3439. https://doi.org/10.1021/ja01190a064

Webb, K. S. (1994). A mild, inexpensive and practical oxidation of sulfides. Tet. Lett., 35, 3457-3460. https://doi.org/10.1016/S0040-4039(00)73209-6

Yu, B., Liu, A. H., He, L. N., Li, B., Diao, Z. F., \& Li, Y. N. (2012). Catalyst-free approach for solvent-dependent selective oxidation of organic sulfides with oxone. Green Chem., 14, 957. https://doi.org/10.1039/c2gc00027j

\section{Copyrights}

Copyright for this article is retained by the author(s), with first publication rights granted to the journal.

This is an open-access article distributed under the terms and conditions of the Creative Commons Attribution license (http://creativecommons.org/licenses/by/4.0/). 